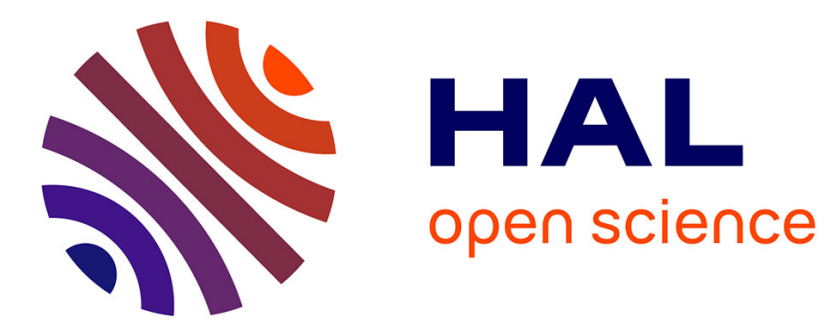

\title{
Les entreprises où les hommes s'exposent à une perte, dans la vue d'un profit Condorcet et l'héritage de d'Alembert
}

Nicolas Rieucau

\section{> To cite this version:}

Nicolas Rieucau. Les entreprises où les hommes s'exposent à une perte, dans la vue d'un profit Condorcet et l'héritage de d'Alembert. Revue Economique, 1998, 49 (5), 10.2307/3502778 . hal01616995

\section{HAL Id: hal-01616995 \\ https://hal.science/hal-01616995}

Submitted on 15 Oct 2017

HAL is a multi-disciplinary open access archive for the deposit and dissemination of scientific research documents, whether they are published or not. The documents may come from teaching and research institutions in France or abroad, or from public or private research centers.
L'archive ouverte pluridisciplinaire HAL, est destinée au dépôt et à la diffusion de documents scientifiques de niveau recherche, publiés ou non, émanant des établissements d'enseignement et de recherche français ou étrangers, des laboratoires publics ou privés. 


\title{
LES ENTREPRISES OU LES HOMMES S'EXPOSENT A UNE PERTE, DANS LA VUE D'UN PROFIT CONDORCET ET L'HERITAGE DE D'ALEMBERT
}

\author{
Nicolas Rieucau* \\ Centre d'Histoire de la Pensée Economique \\ Université Paris I - Panthéon Sorbonne et Université d'Amiens \\ 90, rue de Tolbiac \\ 75634 Paris Cedex 13
}

Classification du JEL : B31 (ou B12)

\begin{abstract}
Au même titre que Voltaire pour ce qui concerne la justice et les lettres et que Turgot en matière d'économie et de politique, d'Alembert est traditionnellement considéré comme le mentor de Condorcet dans les sciences et les mathématiques. Pour autant, l'influence du coéditeur de l'Encyclopédie sur les idées économiques de Condorcet doit-elle être jugée marginale ? Justement pas : en partant d'une conception "mixte » des mathématiques qu'il partage avec d'Alembert et d'une interrogation sur les doutes probabilistes de ce dernier, Condorcet est amené à développer une théorie du choix en univers incertain qui dépasse le domaine classique des jeux de hasard ou des assurances pour s'étendre à celui de l'entreprise économique en tant que telle, qu'elle soit de culture, de commerce ou d'industrie. Ce faisant, il soumet le facteur risque à une formalisation probabiliste sans précédent lorsqu'il envisage le profit perçu par l'entrepreneur.
\end{abstract}

In the same way than Voltaire as far as justice and litterature are concerned, and than Turgot in matter of economics and politics, d'Alembert has been traditionally considered as the mentor of Condorcet in sciences and mathematics. For all that, is the influence of the joint publisher of the Encyclopédie upon Condorcet's ideas in economics to be judged as a marginal one ? Precisely not : departing from a mixed conception of mathematics which he shares with d'Alembert, and from a questionning upon probabilistic doubts of the latter, Condorcet has been induced to develop a choice theory under uncertainty which overtakes the classical field of chance games or of insurances, to extend to the domain of economical enterprise as such, whatsoever consisting in culture, commerce or industry. In doing this, he submits the factor of risk to an unprecedented probabilistic formalisation as he envisages the profit collected by the undertaker.

\footnotetext{
* L'auteur remercie P. Bazard, P. Broda, B. Bru, M. Cohen, P. Crépel, G. Faccarello, A. Lapidus, E. Picavet, P. C. Pradier et G. Rieucau ainsi que l'un des deux rapporteurs anonymes pour leurs remarques critiques. Bien entendu, ils ne sont pas responsables de ce qui pourrait déplaire au lecteur dans ce qui suit.
} 


\section{INTRODUCTION}

Ce qui est communément reconnu comme le legs de Condorcet à l'histoire de la pensée économique renvoie au problème de l'agrégation des préférences individuelles ${ }^{1}$. Le « paradoxe des votes », exhumé par K. J. Arrow $(1951,1963)$ et G. T. Guilbaud (1952), repris notamment par D. Black (1958), et également connu sous le nom d' « effet Condorcet» ou de «paradoxe de Condorcet $»^{2}$, a ainsi marqué de son empreinte la théorie moderne du choix social. En revanche, les travaux relatifs à la théorie du choix non pas collectif mais individuel, ignorent Condorcet, alors qu'ils font référence, à des degrés divers, à nombre de ses collègues géomètres ou philosophes, tels que Buffon, les Bernoulli, Cramer ou encore Laplace. Pourtant, Condorcet semble être le premier écrivain à avoir véritablement usé de «l'art de conjecturer» afin de traiter de la conduite humaine dans certaines de ses activités économiques. A cette occasion, la place qu'il a assignée au risque dans son analyse de la rémunération de l'entrepreneur se compare avantageusement au traitement qu'en proposent ses contemporains.

Afin de mettre en évidence cet apport non négligeable de Condorcet à l'analyse économique, il est indispensable d'effectuer un détour par la conception «mixte », et non pas «pure », que son premier mentor, d'Alembert, développe à l'encontre des sciences mathématiques ; une telle conception permettant en particulier de rendre intelligibles ses doutes sur le calcul des probabilités (section 1). Durant la seconde moitié du XVIII ${ }^{\mathrm{e}}$ siècle, d'Alembert fut incontestablement l'auteur qui afficha le plus de scepticisme sur la valeur scientifique de ce type de calcul ${ }^{3}$. Précisons que cette branche des mathématiques cherchait

\footnotetext{
${ }^{1}$ L'entrée «Condorcet» dans la troisième édition du dictionnaire économique Palgrave (H. Moulin \& H. P. Young (1987)) est à ce titre exemplaire : cette notice traite exclusivement de la théorie des élections de Condorcet.

2 L'expression d' « effet Condorcet» est due à G. T. Guilbaud (1952) qui, le premier, a attiré l'attention sur les travaux fondateurs de Condorcet (et de Borda) concernant les impasses éventuelles du principe majoritaire traditionnel. Dans la première édition de son ouvrage, en 1951, Arrow attribue l'énoncé du paradoxe agrégatif à E. J. Nanson (1883). Chose étrange, Nanson cite pourtant Borda et Condorcet qu'il a dû connaître lui-même grâce à I. Todhunter (1865). Sur cette question, voir G. G. Granger (1956), p. 125.

3 De nombreux commentaires ont récemment été consacrés aux travaux probabilistes de d'Alembert. Nous pensons à E. Yamazaki (1971), L. J. Daston (1979), B. Bru (1983), Z. J. Swijtink (1986) et M. Paty (1987), (1988). Notons au passage que Keynes, dans son Traité des probabilités (1921), évoque à plusieurs reprises les doutes de d'Alembert sur le calcul des probabilités. Son jugement d'ensemble est assez mitigé : « ses opinions sceptiques, rejetées plutôt que réfutées par l'école orthodoxe de Laplace, n'ont pas toujours reçu justice qui leur était due [...] en dépit du caractère erroné d'un grand nombre de ses résultats, [d'Alembert] ne mérite pas pour autant le ridicule dont l'ont affublé certains auteurs, qui acceptèrent sans émettre le moindre doute les conclusions à peine moins incorrectes de la théorie orthodoxe de cette époque », Keynes (1921), p. 398-399,
} 
encore ses marques. Il est ainsi habituellement admis que les premières bases conceptuelles du calcul des probabilités ne furent posées qu'au milieu du XVII ${ }^{\mathrm{e}}$ siècle, à l'occasion de la correspondance entre Fermat et Pascal, et que l'art de conjecturer ne prit finalement l'envergure d'une discipline véritablement constituée qu'en 1812, dans la Théorie analytique des probabilités de Laplace4. En outre, l'extension de l'approche probabiliste en mathématique et la mise en valeur analytique de la notion de moyenne évoluèrent conjointement. Aussi, les remises en cause ou les progrès de la théorie des probabilités au $\mathrm{XVIII}^{\mathrm{e}}$ siècle donnèrent-ils une plus ou moins grande validité scientifique au concept d'espérance mathématique, et inversement. De ce point de vue, les doutes de d'Alembert sur le calcul des probabilités coïncident avec une critique de la « moyenne », entendue comme le produit d'une probabilité et d'une quantité.

Or, la majeure partie de l'œuvre probabiliste de Condorcet peut être interprétée comme une tentative de réponse aux préoccupations de d'Alembert (section 2). Plus précisément, les doutes de d'Alembert constitueront pour Condorcet le levier heuristique, non seulement de sa propre conception des probabilités, mais aussi de la règle de l'espérance qui s'y trouve associée. Par là même, c'est la capacité de cette règle à servir de guide pour le choix en situation d'incertitude que Condorcet cherchera à rétablir. Mais, en s'écartant du scepticisme de d'Alembert, il mettra également en avant une exigence méthodologique : appliquer le calcul des probabilités aux sciences morales, dont l'économie politique. En ce sens, les analyses de d'Alembert, même si elles ne relèvent pas de l'économie, ont exercé une influence considérable sur la pensée économique de son disciple. A ce titre, envisager les nombreux travaux dits d'arithmétique politique de Condorcet, à partir de certaines des réflexions mathématiques de d'Alembert, constitue une entreprise réellement fructueuse exploitée par des historiens des mathématiques comme L. J. Daston (1988, p. 96 et suiv.) et P. Crépel (1988 a). C'est dans cette perspective que nous nous situons afin de mettre en évidence l'étude effectuée par Condorcet du comportement économique en univers incertain 5

notre trad.. P. A. Samuelson (1977), quant à lui, est nettement plus critique. S'appuyant sur l'autorité de I. Todhunter (1865, chpt XIII), il parle en effet des « aberrations » et des « perversités » de d'Alembert.

4 Certes vieilli, l'ouvrage de I. Todhunter (1865) sur l'histoire du calcul des probabilités au XVII ${ }^{\mathrm{e}}$ et au XVIII ${ }^{\mathrm{e}}$ siècle demeure une référence incontournable. Celui, plus récent, de A. Hald (1990) est également remarquable, bien qu'il se limite à la période antérieure à 1750. On peut aussi consulter les contributions de E. Coumet (1970), I. Hacking (1975), B. Sheynin (1977), L. J. Daston (1988) et N. Meusnier (1995).

5 Il est vrai que Condorcet n'emploie pas ces derniers termes. Mais il en exprime néanmoins l'idée à partir du moment où il étudie l'attitude des individus se lançant dans des «entreprises » qui les « exposent à une perte, 
(section 3). Ne se cantonnant pas au thème du jeu de hasard, Condorcet étend en effet sa réflexion au problème des assurances et, plus largement encore, envisage sous un mode probabiliste et «empirique » les spéculations des agents lorsque ceux-ci entreprennent n'importe quel type d'opération économique, qu'elle soit de culture, de commerce ou d'industrie.

\section{D’Alembert : mathématiques mixtes et doutes probabilistes}

D’Alembert est couramment présenté comme un théoricien des «mathématiques mixtes ", discipline dont les objets d'étude ne sont pas de convention, comme ils le sont dans les «mathématiques pures ». Influencé par l'épistémologie de Locke, il considère que la validité de tout résultat mathématique doit, en principe, être estimée d'après sa capacité à rendre compte des phénomènes réels (sur ce point, voir L. J. Daston, 1979). Dans cette optique, d'Alembert n'admet pas que les comportements moraux puissent être appréhendés par le calcul de façon abstraite, en éludant de ce fait les idiosyncrasies des individus et la variété des circonstances dans lesquelles ils sont situés. Certaines de ses réflexions sur le problème de Petersbourg illustrent un tel refus : d'Alembert rejette l'idée que les estimations psychologiques accordées par les individus à telle ou telle quantité monétaire puissent être représentées par une formule hypothétique (1. 1).

De façon presque privilégiée, d'Alembert s'appuie également sur le problème de Petersbourg afin de mettre en valeur ses doutes sur la doctrine des chances. Là aussi, il explique que les résultats traditionnels du calcul, des probabilités en l'occurrence, doivent être évalués, non pas à l'aune de leur exactitude formelle, mais à celle de leur adéquation avec les phénomènes réels. Au début de ses Doutes et questions sur le calcul des probabilités, d'Alembert entend ainsi s'interroger sur la conformité des conclusions théoriques du calcul des probabilités avec le monde réel : «[...] j'admets pour bonne dans la rigueur mathématique, la théorie ordinaire des probabilités, et je vais seulement examiner si les résultats de cette théorie, quand ils seraient hors d'atteinte dans l'abstraction géométrique, ne sont pas susceptibles de restriction, lorsqu'on applique ces résultats à la nature » (d'Alembert, 1767 a, p. 451). A cet égard, la probabilité que l'on assigne a priori à plusieurs événements physiques change nécessairement, à ses yeux, à mesure de leur fréquence d'apparition 
effective. De ce fait, le principe dit de l'équiprobabilité des combinaisons est contesté par le mathématicien français car il ne lui apparaît pas en phase avec la variabilité intrinsèque de la probabilité de ces événements (1. 2). Or, puisque l'expression mathématique d'un tel phénomène demeure selon lui problématique, la probabilité composant l'espérance, et partant, l'espérance elle-même, ne pourraient recevoir d'assise formalisée. Par conséquent, l'espérance ne saurait servir de guide pour la décision puisque, précisément, il serait impossible de l'exprimer formellement.

En la matière, le propos de d'Alembert ne se fait toutefois explicite que lorsqu'il fait état d'un comportement non linéaire 6 des individus face au risque (1. 3). Ce comportement est notamment ${ }^{7}$ mis en avant lorsque, dans ses réflexions sur les jeux de hasard, il explique que les individus, s'ils sont raisonnables, ne vont pas systématiquement s'engager au jeu ou miser en fonction de la règle de l'espérance, la probabilité composant cette dernière étant, selon les cas, « surpondérée » ou « sous-pondérée » par rapport à sa valeur mathématique.

\section{1) La valeur morale de l'espérance : un scandale}

Dans son «Esquisse d'une théorie nouvelle de mesure du sort» (1738), Daniel Bernoulli pose le problème suivant 8 : Pierre joue à croix $(\mathrm{C})$ ou pile $(\mathrm{P})$ avec Paul. Si Pierre obtient $\mathrm{C}$ au premier coup, il doit payer un écu à Paul ; s'il amène $\mathrm{C}$ au deuxième coup, deux écus ; au troisième, quatre écus ; et ainsi de suite - soit $2^{n-1}$ écus si $\mathrm{C}$ vient au bout de $n$

\footnotetext{
probabilités, du reste consacrée à la détermination de la valeur « d'un événement incertain »; BC, p. 387 \& 396. 6 L'adjectif « linéaire » n'est pas employé par d'Alembert dans ses écrits probabilistes mais le fait d'y avoir recours nous semble rendre plus compréhensible son analyse. Rappelons en outre que cet adjectif revêt chez les géomètres du XVIII ${ }^{\mathrm{e}}$ siècle le même sens qu'aujourd'hui.

${ }^{7}$ Le constat d'un comportement non linéaire des individus face au risque est également exposé par d'Alembert dans son analyse du traitement probabiliste d'un problème social, l'inoculation de la petite vérole. Intervient alors dans son analyse ce que nous pourrions qualifier comme étant un problème d' " estimation temporelle des probabilités ». En effet, d'Alembert se demande comment comparer une probabilité représentant un risque faible, mais immédiat - celui de mourir du fait de l'inoculation - à une probabilité relative à un risque élevé, mais éloigné dans le temps - celle de mourir contaminé par la petite vérole ; la première de ces probabilités étant surévaluée par rapport à la seconde. Du même coup, la validité du principe de l'espérance, lorsqu'il s'applique à la durée de la vie, se trouve là encore contestée. Ce doute de d'Alembert ne sera cependant pas analysé ici, dans la mesure où il ne participe pas à l'intelligibilité de la théorie condorcétienne du comportement économique en univers risqué. Voir d'Alembert (1761 b), (1767 b), (1768 c), (1768 e). Pour un commentaire, consulter notamment H. Le Bras (1983).

8 Ce problème, connu par la suite sous le nom de «paradoxe de Saint-Petersbourg », avait été pour la première fois énoncé par Nicolas Bernoulli, cousin de Daniel, dans une lettre adressée à Montmort en 1713. Parmi les contributions récentes relatant l'histoire du problème de Petersbourg, voir P. A. Samuelson (1977), G. Jorland (1987) et J. Dutka (1988).
} 
coups. La question est de savoir combien Paul - qui, sinon, a tout à gagner - doit avancer à Pierre pour que le jeu s'avère équitable. La solution traditionnelle de ce problème est établie d'après l'espérance mathématique du gain de Paul, calculée par l'addition de ses gains successifs multipliés par leur probabilité respective, à savoir : $\left(\frac{1}{2} \times 1\right)+\left(\frac{1}{4} \times 2\right)+\left(\frac{1}{8} \times 4\right)+\cdots+\left(\frac{1}{2^{n}} \times 2^{n-1}\right)+\cdots$, c'est-à-dire $\frac{1}{2}+\frac{1}{2}+\frac{1}{2}+\cdots+\frac{1}{2}+\cdots$.

La mise de Paul devra ainsi être de $\frac{1}{2}$ multiplié par l'infini, soit $\sum_{n=1}^{\infty} \frac{1}{2^{n}} 2^{n-1}$, puisque on ne voit pas pourquoi il doit être fixé un nombre maximal de coups, c'est-à-dire le moment où $\mathrm{C}$ adviendra. Mais il est bien évidemment absurde que Paul mise une somme infinie pour faire jeu équitable avec Pierre...

Face à cette impasse logique, D. Bernoulli, mais aussi Cramer (1728) et Buffon (1730), proposent de remplacer le caractère mathématique de l'espérance par son estimation psychologique, morale 9 , variable selon la grandeur initiale de la fortune des joueurs. Dans cette perspective, l'enjeu de Paul doit être évalué d'après ce que les économistes appelleront plus tard l'utilité marginale de son revenu. Par exemple, si Paul dispose d'un revenu initial de 100, il n'acceptera pas de le miser tout entier pour avoir une probabilité $\frac{1}{2}$ d'en gagner 200 puisque les 100 unités supplémentaires de revenu dont il bénéficierait lui procureraient une satisfaction inférieure aux 100 premières unités correspondant à sa fortune initiale. Le revenu de départ de Paul marque donc une limite supérieure à l'utilité qu'il retire du jeu et garantit, du même coup, une mise dont le montant sera fini.

La critique que d'Alembert formule à l'encontre de ce type de résolutions peut être appréhendée à partir de la distinction terminologique qu'il reprend de Fontenelle (selon T. L. Hankins, 1970, p. 94), entre esprit géomètre et esprit géométrique. L'esprit géomètre est assimilé au talent pour les mathématiques pures, tandis que l'esprit géométrique se ramène à une réflexion sur ses conditions d'application à d'autres domaines. Comme d'Alembert l'explique dans ses Eléments de philosophie, exercer un esprit géomètre en dehors des mathématiques, pour traiter des phénomènes moraux en l'occurrence, et ce, sans user de l'esprit géométrique, reviendrait à « imaginer que l'essence des démonstrations consistât dans la forme géométrique » (d'Alembert, 1759, p. 34-35. Voir également d'Alembert, 1757, p. 628). De là, il s'en prend à ces «quelques Philosophes » ayant appliqué sans vergogne les

\footnotetext{
${ }^{9}$ Cramer parle à ce titre d' « espérance morale »; Cramer (1728), p. 561. Cette expression sera popularisée par Laplace (1812), livre 2, chpt X.
} 
mathématiques à n'importe quel sujet et croyant que « raisonner en forme, c'était raisonner juste » et leur reproche notamment d'avoir été jusqu'à mettre « des figures de géométrie dans des traités de l'âme » (d'Alembert, 1759, p. 35).

De ce même point de vue, on pourrait affirmer que, d'après d'Alembert, l'esprit géométrique a fait défaut aux philosophes qui ont proposé des solutions de nature subjective au problème de Petersbourg. Aussi, dans son article Croix ou Pile, d'Alembert qualifie-t-il de véritable «scandale » (d'Alembert, 1754, p. 513) ce type de résolutions. Certes, précise-t-il, « il est certain, par exemple, que de deux hommes inégalement riches qui jouent à jeu égal suivant les règles ordinaires, celui qui est le moins riche risque plus que l'autre » (ibid.) ${ }^{10}$. Mais, particulières à chaque individu et variant au gré des circonstances, les considérations morales ne sauraient recevoir de formalisation univoque et hypothétique. Conformément à sa conception appliquée, mixte, des sciences mathématiques, d'Alembert estime ainsi que l'espérance morale devrait être idéalement estimée par une multiplicité d'expressions, chacune d'entre elles faisant état des personnalités propres aux agents et des circonstances variables dans lesquelles ils sont placés (voir aussi d'Alembert, 1761 a, p. 24-25).

Par ailleurs, on remarque que dans ses Doutes et questions sur le calcul des probabilités, d'Alembert dresse un rapprochement entre cette diversité des phénomènes moraux et celle afférente aux événements physiques : « il n'est pas dans la nature qu'un effet soit toujours et constamment le même, comme il n'est pas dans la nature que tous les hommes et tous les arbres se ressemblent» (d'Alembert, 1767 a, p. 454). Or, cette diversité des événements physiques, qu'il estime intrinsèque, lui permet justement d'énoncer l'un de ses doutes majeurs à l'encontre du calcul des probabilités, sur lequel nous allons maintenant nous attarder.

\section{2) La critique de l'équiprobabilité des combinaisons}

\footnotetext{
10 Par « jeu égal», d'Alembert entend ici une situation où les espérances mathématiques de gain des joueurs sont équivalentes à leur mise. L'analyse que Condorcet effectue de cette notion est présentée plus loin dans notre texte.
} 
Au début de ses Réflexions sur le calcul des probabilités, d'Alembert prend le cas où c'est Pierre et non Paul qui doit miser, et il explique à cette occasion que le caractère infini de l'espérance de Pierre est une hypothèse « absurde » puisque $\mathrm{C}$ doit nécessairement survenir à la suite d'un nombre de lancers fini. Par suite, «l'espérance de Pierre n'est que finie » (d'Alembert, 1761 a, p. 3). Certes, son raisonnement peut sembler critiquable car c'est précisément parce que l'on ne connaît pas le moment où $\mathrm{C}$ adviendra - et donc le nombre maximum de coups - que l'on suppose un nombre de lancers infini. Mais il ne faut pas perdre de vue que d'Alembert entend cantonner son propos dans un cadre physique et non pas mathématique, dans la mesure où il invoque la nécessité d'évaluer les résultats du calcul des probabilités d'après leur capacité à rendre compte de l'existence des événements réels. Il estime par conséquent naturel de borner l'espérance mathématique de Pierre puisque, sous un angle physique, $\mathrm{C}$ doit forcément arriver à un moment ou à un autre.

Le même type de raisonnement sous-tend une objection, plus radicale, que d'Alembert mentionne un peu plus loin dans son texte : au lieu de considérer les résultats des différents lancers comme équiprobables, il convient d'évacuer certaines combinaisons mathématiquement (ou métaphysiquement ${ }^{11}$ ) envisageables, c'est-à-dire faisant intervenir $\mathrm{C}$ ou $\mathrm{P}$ un grand nombre de fois de suite, au profit de combinaisons non uniformes, physiquement plus vraisemblables, attendu que « la variété des événements successifs est un phénomène constant de la nature ; et que leur similitude constante ou répétée un grand nombre de fois, est au contraire un phénomène qui n'arrivera jamais » (ibid., p. 15) ${ }^{12}$. Cette objection est bien sûr arbitraire mais là encore, il convient de garder à l'esprit que d'Alembert prétend situer sa réflexion d'un point de vue physique et non pas mathématique. C'est pourquoi, $n$ étant un nombre élevé, si l'arrivée de $\mathrm{P} n$ fois de suite est mathématiquement possible, cette éventualité se dissout à l'aune de l'ordre physique des choses. De ce même point de vue, la probabilité d'obtenir $\mathrm{C}$ au bout de tel ou tel nombre de coups est donc conditionnée par les résultats obtenus aux lancers précédents. L'équiprobabilité des combinaisons se trouve par là même contestée car, à mesure que $\mathrm{P}$ survient sans manquer, sa

\footnotetext{
11 Le sens général du texte indique en effet que «métaphysiquement possible» est ici synonyme de «mathématiquement possible». Dans ses Doutes et questions sur le calcul des probabilités (1767 a), d'Alembert privilégiera d'ailleurs la seconde de ces expressions pour signifier la première.

12 De toute façon, puisque le jeu doit s'interrompre lorsque $C$ survient, on voit mal comment il pourrait arriver ne serait-ce que deux fois de suite ! D'Alembert avait déjà fait cette remarque, qu'il ne reprend pas ici, dans son article Croix ou Pile, et ce, alors qu'il examinait le cas où les règles du jeu limitent la partie à deux lancers. Voir d'Alembert (1754), p. 513.
} 
probabilité de réalisation au lancer suivant s'éloigne de $\frac{1}{2}$ pour tendre progressivement vers 0 .

Or d'Alembert affichera constamment un certain scepticisme quant à l'idée de formaliser sa croyance en la décroissance progressive de la probabilité ${ }^{13}$ et, au terme du quatrième volume de ses Opuscules mathématiques, il présentera sa mise en cause de l'équiprobabilité des combinaisons comme 1' « une des plus fortes objections qu'on puisse faire contre les règles reçues de l'analyse des jeux » (d'Alembert, $1768 \mathrm{~d}, \mathrm{p} .304)^{14}$. Du même coup, la valeur praxéologique de l'espérance se trouve contestée. En effet, en critiquant le principe de l'égale possibilité des combinaisons, d'Alembert s'en prend également à la formalisation de leur probabilité respective, probabilité qui compose précisément l'espérance. Cependant, la mise en cause de la capacité de l'espérance à guider la conduite humaine n'est pas, ici, explicitement exprimée par d'Alembert. Cela n'est pas le cas lorsqu'il développe le second de ses doutes sur la doctrine des chances qu'il nous reste à examiner. Cette fois, la capacité de la règle de l'espérance à guider le comportement est clairement récusée. Et, si la théorie ordinaire des probabilités se trouve là encore sur la sellette, compte tenu de son inaptitude à rendre compte des phénomènes réels, ces derniers sont envisagés dans leur nature morale et non pas physique.

\section{3) Un comportement non linéaire face au risque}

13 «Assigner la loi de cette diminution, c'est ce que ni moi, ni personne, je crois, ne peut faire»; d'Alembert (1767 a), p. 458. Voir aussi d'Alembert (1761 a), p. 15. Et si, dans le volume IV (1768 a, p. 75) et le volume VII (1780, p. 50 et suiv.) de ses Opuscules, d'Alembert suggère pourtant certaines formules mathématiques rendant compte de l'affaiblissement de cette probabilité, il va de soi qu'il a alors pleinement conscience de leur caractère arbitraire, comme le souligne M. Paty (1988).

14 Si l'on raisonne en temps discret, comme c'est le cas dans le jeu de Croix ou Pile, on peut considérer que cette critique de l'équiprobabilité des combinaisons repose sur l'idée que la probabilité varie à mesure que le temps s'écoule, idée dont d'Alembert doute qu'elle puisse être formalisée. Si l'on raisonne maintenant en temps continu, le fait que la probabilité puisse varier dans le temps pose le difficile problème des «combinaisons chronologiques », tel que le qualifient les mathématiciens contemporains. Ce problème, et les doutes conséquents sur sa formalisation, ne sont pas explicitement posés par d'Alembert, même s'ils découlent logiquement de son raisonnement. Il est cependant remarquable que Condorcet en propose un traitement mathématique dans la «Troisième partie » (1785) de son Mémoire sur le calcul des probabilités. Sur ce point relativement technique, voir P. Crépel (1988 b). 
Examinant le jeu de Petersbourg dans son article Croix ou Pile, d'Alembert explique que, dans la mesure où la fortune de Pierre est nécessairement finie, l'espérance mathématique et donc la mise de Paul doivent l'être également, puisque Pierre ne pourrait lui verser une somme dépassant sa fortune initiale. D'Alembert prend le cas où la fortune de Pierre serait de $2^{20}$ écus. Le jeu se trouve par conséquent limité à 21 coups, puisque l'espérance et la mise de Paul ne doivent pas excéder $\sum_{k=1}^{20} \frac{1}{2^{k}} 2^{k-1}$, sans quoi Pierre ne serait pas en état de le payer (d'Alembert, 1754, p. 513).

Non sans reconnaître le caractère « ingénieux » de cette solution, d'Alembert la remet cependant immédiatement en cause car détachée de l'attitude réelle des joueurs, à supposer qu'ils soient des êtres sensés : Paul «serait un fou» de miser une somme aussi grande, correspondant pourtant à son espérance, « mais il ne le serait, que parce que Pierre est un fou aussi de proposer un jeu où lui Pierre peut perdre en une minute des sommes immenses » (ibid.) ${ }^{15}$. Ainsi, l'espérance résultant du produit d'un gain élevé et d'une probabilité faible en l'occurrence la probabilité que $\mathrm{P}$ survienne au $21^{\mathrm{e}}$ lancer - ne sert pas de guide à la conduite de joueurs raisonnables et prudents : «[...] les règles de probabilité sont en défaut lorsqu'elles proposent, pour trouver l'enjeu, de multiplier la somme espérée par la probabilité du cas qui doit faire gagner cette somme ; parce que, quelque énorme que soit la somme espérée, la probabilité de la gagner peut être si petite, qu'on serait insensé de jouer un pareil jeu » (d'Alembert, 1767 a, p. 453. Voir aussi d'Alembert, 1761 a, p. 8).

La nécessité de négliger les petites probabilités est également invoquée par d'Alembert lorsqu'il s'agit de comparer des espérances mathématiquement équivalentes, l'une d'elles étant composée d'une chance de gain proche de la nullité tandis que l'autre est associée à une probabilité située au voisinage de la certitude. D'Alembert prend par exemple le cas d'un joueur ayant à choisir entre une probabilité $\frac{99}{100}$ de gagner 1000 écus et une probabilité $\frac{1}{100}$ de gagner 99000 écus. Dans les deux cas, son espérance mathématique est de 990 écus mais «quel sera l'homme assez insensé pour préférer celle qui donnera 99 mille écus ? L'espérance dans les deux cas n'est pas réellement la même, quoi qu'elle soit la même suivant les règles des probabilités » (d'Alembert, 1768 b, p. 83).

15 Z. G. Swijtink (1986) rapporte à cet égard que les adeptes du jeu de Croix ou Pile dans les salons parisiens ne misaient pas plus de 10 ou 20 écus. 
Cette critique des vertus praxéologiques de la règle traditionnelle de l'espérance s'étend également aux situations où, bien qu'aucune des chances de gain ne soient faibles, l'une d'elles est égale à la certitude. D'Alembert explique ainsi qu'un joueur préfére être certain de gagner 500 livres plutôt que d'avoir une probabilité $\frac{1}{2}$ d'en gagner 1000 . Sa démonstration vérifie en somme l'adage populaire Un bon «tiens » vaut mieux que deux «tu l'auras ». Dans cette optique, d'Alembert estime que son raisonnement mène à une remise en cause de la théorie du hasard en tant que telle, lorsque celle-ci conçoit la probabilité comme une «fraction de la certitude», car la probabilité comporte par définition un risque, ce qui n'est pas le cas pour la certitude. En ce sens, «mille probabilités ne feront jamais une certitude » (ibid. Voir aussi d'Alembert, 1783 b, MS 1793, f. 373) ; d'Alembert mettant en évidence ce que nous reconnaîtrions aujourd'hui comme un principe de sous-additivité des probabilités 16 , lié ici à une minoration psychologique des probabilités inférieures à la certitude quand elles sont mises en rapport avec une perspective certaine. Cette discontinuité entre une probabilité - quelque grande qu'elle fût - et la certitude, n'est pas non plus sans évoquer le phénomène de «préférence pour la sécurité » mis en évidence par M. Allais (1953), phénomène également qualifié d' «effet de certitude » depuis les travaux de D. Kahneman et A. Tversky (1979).

En outre, les exemples précédents indiquent que les individus se focalisent non sur le gain associé à l'espérance mais sur son élément aléatoire. Aussi, d'Alembert affirme-t-il que «c'est principalement la probabilité qui doit régler l'espérance » (d'Alembert, 1768 b, p. 8283), le gain ne devant être considéré «que d'une manière subordonnée au degré de probabilité » (ibid., p. 83). Si l'on s'autorise un dernier rapprochement avec la théorie moderne de la décision, on fera remarquer que le phénomène exposé par d'Alembert n'est pas sans rappeler l'un de ceux que P. Delquié (1993) choisit de ranger sous le vocable de «biais de prééminence » dû, en l'occurrence, à la considération prioritaire accordée dans certains cas à la probabilité composant l'espérance ${ }^{17}$. Ce privilège attaché à la probabilité se traduit en dernière analyse par un comportement non linéaire des individus face au risque, dans la mesure où ils affichent une préférence pour les probabilités équivalentes à la certitude ou situées à son voisinage, et négligent au contraire celles qui sont de faible valeur.

\footnotetext{
16 A ce sujet, voir J. Quiggin (1982), M. E. Yaari (1987) ou A. Tversky \& D. Kahneman (1992).

17 Sur ce point, voir également M. Allais (1953), D. Kahneman \& A. Tversky (1979) et K. R. MacCrimmon \& S. Larsson (1979).
} 
Il n'empêche que la négligence des petites probabilités - en supposant même que leurs estimations subjectives sont uniformes - est également, d'après d'Alembert, non formalisable. En effet, lorsqu'il énonce sa critique de l'équiprobabilité des combinaisons, rappelons que d'Alembert doute qu'il soit possible d'offrir un traitement mathématique satisfaisant de la probabilité décroissante affectant un événement à mesure que celui-ci est survenu $n$ fois de suite dans le passé. Or, dans ses Réflexions sur le calcul des probabilités, il reproduit une opinion similaire lorsqu'il revendique la nécessité de considérer comme nulles les faibles probabilités à partir d'un certain seuil et de modifier en conséquence les règles traditionnelles de la théorie du hasard : «quel est le terme où la probabilité commence à pouvoir être regardée comme nulle ? ${ }^{18}$. En supposant même que l'on puisse fixer ce seuil, il se demande si les probabilités qui lui sont juste supérieures doivent être considérées comme plus faibles que leur valeur mathématique. Si tel n'est pas le cas, d'Alembert estime irrecevable de passer «brusquement et sans gradation, d'une expression finie à une valeur nulle». Dans le cas contraire, on retombe sur les difficultés de construction d'une « loi de diminution de la probabilité » déjà présente dans la remise en cause de l'équiprobabilité des combinaisons : « et s'il faut regarder ces probabilités comme plus petites qu'elles ne sont, je demande suivant quelle loi il faut les diminuer »(d'Alembert, 1761 a, p. 11). D'Alembert ajoute que, à supposer même qu'il soit possible d'évaluer correctement une telle loi de diminution, se pose de surcroît la question du terme jusqu'auquel il conviendrait de l'appliquer (ibid.).

Ne pouvant être formalisée, l'idée selon laquelle les petites probabilités doivent être considérées comme nulles dans la pratique est donc scientifiquement stérile pour d'Alembert. Il rejette du même coup l'idée de «probabilité morale» considérée par Buffon comme équivalente à zéro lorsqu'elle atteint une valeur inférieure ou égale à $\frac{1}{10000}$ (Buffon, 1777, p. 37-39) ${ }^{19}$; idée aujourd'hui reconnue par les historiens des mathématiques comme le « principe de Buffon-Cournot » ou « principe de Cournot » 20 .

\footnotetext{
18 Dans leur correspondance, N. Bernoulli et Cramer s'étaient déjà heurtés à ce type de réflexion, sans s'y attarder toutefois aussi longuement que d'Alembert. Voir Lettre de N. Bernoulli à Cramer, 3 juil. 1728, p. 562 ; Lettre de Cramer à N. Bernoulli, 27 sept. 1728, p. 564.

19 Par parenthèse, notons que Morgenstern (1974) s'oppose à ce genre d'argument car il estime que les capacités intellectuelles des individus ne leur permettent pas de percevoir la valeur exacte des très petites probabilités. De ce fait, Morgenstern dénie toute pertinence aux tests empiriques qui soumettent aux individus de telles probabilités et mènent justement à une remise en cause de son modèle d'« utilité espérée ».

20 M. Fréchet, à qui l'on doit l'expression de « principe de Buffon-Cournot », affirme, sans citer de texte précis, que ce principe est «déjà dans la pensée de d'Alembert»; M. Fréchet (1955), p. 208. S'appuyant sur les Réflexions sur le calcul des probabilités (1761) de d'Alembert, M. Allais (1979 b) choisit pour sa part de
} 
Par ailleurs, on comprendra aisément que d'Alembert récuse aussi la réciproque de la notion de «probabilité morale nulle» développée par Buffon, à savoir celle de «certitude morale » 21 , c'est-à-dire l'idée selon laquelle une très grande probabilité doit être considérée comme équivalente à la certitude. Certes, il ne s'interroge pas sur la formalisation d'une quelconque « loi d'augmentation de la probabilité » - il aurait pu d'ailleurs le faire, attendu que, conformément à sa critique de l'équiprobabilité des combinaisons, un événement voit ses chances de réalisation augmenter à mesure qu'il n'est pas survenu dans le passé. Mais, de façon plus simple, on rappellera que d'Alembert se refuse à assimiler une probabilité, aussi grande soit-elle, à une certitude qui elle, ne comporte pas de risque.

Cette critique de l'espérance mathématique développée par d'Alembert lorsqu'il considère les jeux de hasard débouche ainsi sur un certain nombre de réquisits non formalisables à ses yeux. Aussi, l'impératif de prudence, dicté selon lui par le simple bon sens, n'est-il pas rigoureusement axiomatisé dans son analyse. En d'autres termes, l'attitude non linéaire des joueurs face au risque, en faisant même abstraction de leur subjectivité particulière, ne saurait se soumettre à la fermeté du calcul.

D'Alembert n'a pas manqué d'interpeller ses collègues géomètres au sujet de ses mises en cause, qu'il estimait fondamentales, de l'art de conjecturer. On lit par exemple, à la fin du quatrième volume de ses Opuscules mathématiques : «mais j’espère aussi que mes doutes engageront d'habiles gens sans préjugés à approfondir cette matière épineuse, et à lui donner le degré d'évidence dont elle peut être susceptible » (d'Alembert, 1768 d, p. 308)22. Nous allons voir que Condorcet ne restera pas insensible à ce genre d'appel d'offre scientifique exprimé par l'esprit dominant alors les mathématiques françaises.

qualifier « principe de d'Alembert » une telle idée et regrette, que du point de vue « déontologique », Buffon ne fasse pas référence à d'Alembert; M. Allais (1979 b), p. 658-659. Allais et Fréchet nous semblent commettre une double erreur : non seulement d'Alembert se démarque de Buffon puisqu'il refuse de formaliser ce principe mais, de plus, les travaux de l'auteur de l'Histoire naturelle sur le sujet sont antérieurs aux siens : l'Essai d'arithmétique morale a certes paru en 1777 mais, comme le signale J. Roger (1977), cet ouvrage est en fait un patchwork de textes anciens, pour la plupart inédits. Même si J. Roger n'en suggère pas la datation exacte, le passage où Buffon expose la notion de « probabilité morale nulle » a en tout cas été écrit avant 1751 car nous en avons trouvé une allusion dans l'ajout de Diderot à l'article Absent de d'Alembert. Voir Diderot (1751), p. 4041.

21 Cette expression est relativement répandue lorsque Buffon l'emploie pour son propre compte. Dans son Introduction à la philosophie (1736, p. 128), notons que 's Gravesande estime qu'elle est d'usage « vulgaire ». O. B. Sheynin (1977) rapporte à cet égard qu'on la trouve déjà chez des auteurs tels que Descartes, Huyghens, Leibniz ainsi que chez Jean et Nicolas Bernoulli.

22 On peut également se contenter de citer l'intitulé de l'un de ses principaux textes consacré à la théorie du hasard : Doutes et questions sur le calcul des probabilités (1767 a). 


\section{Condorcet face à d'Alembert}

Dans une lettre adressée à Garat vers 1784-85, Condorcet accorde un certain crédit intellectuel aux réflexions probabilistes de d'Alembert : «M. d'Alembert a donné aussi sur les principes fondamentaux de ce calcul [des probabilités] des réflexions qui ont fait sentir la nécessité de ne les employer qu'avec précautions » (BC, p. 542). De même, à l'occasion de l'éloge qu'il consacre à son mentor en 1784, Condorcet reconnaît que si le calcul des probabilités « s'appuie un jour sur des bases plus certaines, c'est à M. d'Alembert que nous en aurons l'obligation » (OC, t. III, p. 92). Cependant, Condorcet aura précisé auparavant : «L'opinion de M. d'Alembert a le danger de trop resserrer le champ où l'esprit humain peut s'exercer ; de rendre l'ignorance présomptueuse, en lui montrant ce qu'elle ne connaît pas comme impossible à connaître » (ibid., p. 79). Il semble ne pas faire de doute que Condorcet épingle ici le scepticisme, excessif à ses yeux, qui émane des interrogations de d'Alembert sur l'art de conjecturer.

De fait, dans certains de ses manuscrits de la décennie 1770 et, plus encore, dans ses mémoires mathématiques des années 1780 , Condorcet cherche à rétablir ou à amender les «bases » du calcul des probabilités 23 . Certes, comme d'Alembert, il demeure dans une certaine mesure un théoricien des mathématiques mixtes. A ce titre, Condorcet s'interroge sur la validité de l'application du calcul, en particulier des probabilités, aux phénomènes réels. Cette démarche ne doit pas pour autant mener, selon lui, à «rendre l'ignorance présomptueuse », c'est-à-dire à dénier toute portée scientifique à l'art de conjecturer. Dans cette optique, Condorcet réhabilite le principe de l'équiprobabilité des combinaisons critiqué par d'Alembert : si ce principe n'est que la marque de notre ignorance des événements réels, rien n'empêche, tout en l'envisageant comme une pure considération intellectuelle, d'établir des spéculations à partir de celui-ci. Du même coup, nous verrons que le clivage posé par

\footnotetext{
23 Remarquons cependant que Condorcet ne parviendra pas à répondre au doute de d'Alembert concernant le traitement probabiliste du problème de l'inoculation de la petite vérole. Certes, il défend dans de nombreux textes publiés la pratique de l'inoculation en elle-même mais à aucun endroit son propos n'est soutenu par une quelconque argumentation et, s'il évoque parfois le problème de l'" estimation temporelle des probabilités » posé par d'Alembert, il ne cherche à le résoudre en aucune manière. Voir Eloge de la Condamine (1774), OC, t. II, p. 190-196, Eloge de Lieutaud (1781), OC, t. II, p. 407, Eloge de Tronchin (1781), OC, t. II, p. 503-504, Eloge de D. Bernoulli (1782), OC, t. II, p. 562-563, Eloge de d'Alembert (1784), OC, t. III, p. 93-95 et enfin Eloge de Bouvart (1787), OC, t. III, p. 280 \& 285. Dans ses manuscrits, les rares fois où il tente de justifier « analytiquement» une telle pratique, Condorcet se montre d'ailleurs très hésitant. Dans son premier texte consacré au calcul des probabilités, en 1770, le passage relatif au thème de l'inoculation est ainsi barré dans sa majeure partie. Il en est de même dans un manuscrit plus tardif, concernant l'arithmétique politique. Voir Z 30, f. 6 ; BC, p. 261 et MS 855, f. 178-179; BC, p. 340-341.
} 
Condorcet entre la réalisation d'un phénomène et sa mathématique lui permet de dresser sa théorie dite du motif de croire, soutien scientifique de son désir d'appliquer le calcul des probabilités aux autres champs de la connaissance, dont l'économie (2. 1). Cela étant, à l'instar de d'Alembert, Condorcet se refuse à envisager les comportements moraux à partir d'un mode de calcul univoque et abstrait (2. 2). Si la représentation morale de l'espérance est à cet égard récusée, Condorcet répugne-t-il également à recourir à son estimation mathématique ? Justement pas : en l'envisageant comme une règle valable lorsque le nombre d'épreuves jouées est élevé, il tente de dissiper les doutes que d'Alembert exprime à son encontre dans ses réflexions sur les jeux de hasard (2.3). Cependant, tout comme d'Alembert, Condorcet envisage la contradiction éventuelle pouvant intervenir entre la règle de l'espérance mathématique et l'impératif de prudence. Un cas typique illustrant cette contradiction survient lorsque les individus, risquant la ruine, ne sont pas certains de pouvoir jouer un nombre suffisamment grand d'épreuves pour être assurés de gagner une somme correspondant à leur espérance.

\section{1) Reconnaissance de l'équiprobabilité des combinaisons et théorie du motif de croire}

Dans l'un de ses premiers manuscrits probabilistes, rédigé vers 1773-74, Condorcet part de l'exemple du jet de dés où il s'agit d'obtenir deux six. Avant le lancer, la probabilité d'obtenir deux six est de $\frac{1}{36}$. Or, si l'on recouvre d'un voile les deux dés une fois qu'ils ont été lancés, cette probabilité de découvrir deux six demeure de $\frac{1}{36}$. La probabilité de réalisation d'un événement futur (va-t-on obtenir deux six ?) est donc la même que celle d'un événement passé (a-t-on effectivement deux six sous le voile ?). Aussi, une interrogation sur la conformité du calcul probabiliste avec l'ordre des phénomènes réels n'est-elle déniée ici que parce qu'elle apparaît comme vide de sens puisque la probabilité de réalisation d'un événement est indépendante de son apparition effective (les dés sous le voile peuvent ou non révéler deux six). Cette probabilité de réalisation est, par suite, « une considération purement intellectuelle qui n'a aucun rapport par elle-même avec l'état physique des choses » (MS 875, f. 90-91 ; BC, p. 289) ${ }^{24}$. De là, Condorcet insiste sur le fait que notre connaissance du monde extérieur ne peut être que probable car elle se fonde sur l'hypothèse selon laquelle ce qui est 
survenu dans le passé continuera de se présenter dans le futur selon la même régularité. Le nombre de fois où un événement a été observé conditionne donc l'estimation de sa probabilité d'existence à venir ; estimation qui n'a rien à voir avec l'ordre physique des choses. Dans ce manuscrit de 1773-74, Condorcet précise que les connaissances morales, relatives à la conduite humaine au sens large, sont de la même nature que les connaissances sur le monde physique : probables. Dans les deux cas en effet, les certitudes que nous établissons se fondent sur notre expérience passée et reposent donc sur l'hypothèse selon laquelle ce qui est advenu jusqu'à maintenant continuera de se réaliser dans le futur (MS 875, f. 95-97 ; BC, p. 292-293).

Ce manuscrit de 1773-74 préfigure la doctrine du «motif de croire » de Condorcet, fondement de sa théorie de la connaissance et partant, des prescriptions méthodologiques qui en émanent. L'exposé de la maturation progressive de cette doctrine, qui intègre la loi dite de Bayes-Laplace ${ }^{25}$, ne nous occupera pas ici (à ce sujet, voir par exemple K. M. Baker, 1975, p. 232 et suiv.). Contentons-nous simplement de remarquer qu'elle trouve seulement son expression pleine et entière dans plusieurs textes publiés par Condorcet vers le milieu des années $1780^{26}$. L'exemple démonstratif privilégié alors par l'encyclopédiste est celui de l'urne : dans une urne composée de boules noires et de boules blanches, si l'on a tiré dans le passé, en les remettant, $n$ boules noires et $m$ boules blanches, la probabilité d'obtenir au tirage suivant une boule noire, plutôt que d'être estimée par sa simple fréquence, doit être évaluée selon Condorcet par le rapport $\frac{n+1}{m+n+2}$. Ce rapport, qui fonde notre motif de croire en l'occurrence future d'une nouvelle boule noire, repose sur l'hypothèse d'une régularité dans les phénomènes de la nature ; phénomènes dont l'ordre exact demeure inconnu et que nous supposons, pour cette raison même, comme régulier. L'équiprobabilité, marque de notre ignorance, se trouve du même coup réhabilitée : «l'égale possibilité des événements [n'est] pour nous que l'ignorance absolue des causes qui peuvent déterminer un événement plutôt qu'un autre » (Eléments, 1786-87, OB, p. 537). Or, en rétablissant le principe de l'équiprobabilité, Condorcet accorde une véritable crédibilité heuristique à l'art de

\footnotetext{
24 Idée déjà exprimée par 's Gravesande dans son Introduction à la philosophie $(1736$, p. 130) et surtout par Hume dans la troisième partie du «Livre premier » (1739) du Traité de la nature humaine dont K. M. Baker (1975, p. 182 et suiv.) estime que Condorcet s'est grandement inspiré.

25 Nous préférons parler de loi «dite» de Bayes-Laplace car il est possible que Condorcet ait découvert ce principe mathématique, au début des années 1770, indépendamment de ces deux auteurs. Sur ce point, voir P. Crépel (1988 b).
} 
conjecturer. Celui-ci n'est effectivement «qu'un supplément à notre ignorance des événements réels, des lois réelles observées dans la nature » (Probabilité, 1785, BC, p. 515) ; et, dans cette perspective, il doit notamment permettre d'évaluer le degré de certitude des propositions relatives aux phénomènes moraux et politiques, c'est-à-dire le motif que nous avons de les croire.

Cette exigence méthodologique éclaire à cet égard la signification particulière que Condorcet accorde à l' " arithmétique politique » qui désigne ordinairement le dénombrement des habitants, l'estimation de leur durée de vie, le calcul des chiffres de la balance du commerce, de la production et de la consommation nationale, de la quantité des revenus distribués, des impôts etc. Ses travaux en la matière se font en effet sous le couvert d'une conception plus étendue de cette discipline, qu'il définit comme «l'application du calcul aux sciences politiques »(Arithmétique politique, début des années 1780, BC, p. 483). Un tel élargissement dérive essentiellement du rôle primordial assigné par Condorcet au calcul des probabilités $^{27}$ : à l'aide de la loi dite de Bayes-Laplace, sa théorie du motif de croire lui permet d'intégrer, ou du moins de systématiser la place de nouveaux éléments et, surtout, de les appréhender de façon véritablement scientifique. Les calculs de loteries, d'annuités, de rentes viagères, d'assurances, de droits de succession, de modes d'élection ou de délibération sont ainsi considérés comme des objets d'étude à part entière de l'arithmétique politique 28 .

On remarque cependant qu'une telle « application du calcul aux sciences politiques » ne s'est pas concrétisée, dans l'œuvre de Condorcet, par l'idée de représenter mathématiquement la procédure du marché grâce à un raisonnement hypothétique sur les comportements d'offre et de demande. Pourtant, lorsqu'il ébauche, en 1793, les grandes

26 A savoir, principalement, l'article Probabilité (1785), le Discours préliminaire de l’Essai (1785) et les Eléments (1786-87).

27 Dans un fragment manuscrit intitulé Arithmétique politique (1772), Condorcet va même jusqu'à faire du calcul des probabilités le critère distinctif de deux définitions de cette discipline. Ainsi, « on peut entendre par là deux choses [ : ] ou l'exposition exacte du nombre des hommes, de leur durée de vie [...] c'est purement une science de faits, ou bien on entend ce que, posé les faits établis par les calculs dont on vient de parler, on doit se conduire pour les événements futurs d'après le calcul des probabilités »; MS 873, f. 66-67; BC, p. 303. Dans ses écrits ultérieurs, Condorcet s'inspirera de la même démarche lorsqu'il présentera les trois parties de l'arithmétique politique telle qu'il la conçoit $: 1^{\circ}$ détermination des faits, $2^{\circ}$ résultats des faits, $3^{\circ}$ probabilité des faits et des résultats. Voir Réflexions sur l'arithmétique politique (vers 1780), Z 50 ; BC, p. 337 ; Arithmétique politique (1784), BC, p. 483. Condorcet réitérera une idée similaire lorsqu'il évoquera les trois parties principales de la méthode sous-jacente à sa « Mathématique sociale ». Voir Tableau général (1793), OC, t. I, p. 545.

28 A l'exception de l'Essai (1785) et des Eléments (1786-87), on trouvera dans B. Bru \& P. Crépel (1994) une édition critique et commentée de l'ensemble des travaux d'arithmétique politique de Condorcet effectués entre 1767 et 1789. Pour une présentation de ceux effectués pendant la période révolutionnaire, voir P. Crépel (1990). 
lignes de son projet de « Mathématique sociale », dont la théorie du motif de croire - jointe à une signification mathématique accordée à l'analyse (sur ce point, voir K. M. Baker, 1967 ; 1975, p. 151-155) - constitue la clef de construction, Condorcet précise que cette nouvelle discipline recouvre «l'économie politique presque entière » (Tableau général, OC, t. I, p. 572). La raison de ce paradoxe est facile à élucider : ainsi que nous allons le voir, Condorcet refuse d'appréhender les phénomènes moraux à partir d'une formalisation abstraite et ce, à l'instar de d'Alembert.

\section{2) Le refus d'une mesure hypothétique des comportements moraux}

Si la foi de Condorcet en l'application de l'analyse mathématique aux autres champs de la connaissance fait corps avec son œuvre, il n'empêche que le marquis lui donne certaines limites. A cet égard, il récuse, comme d'Alembert, l'idée de spécifier par une formule hypothétique les comportements moraux. Il est vrai que Turgot, le mentor de Condorcet en politique, affiche également une certaine réticence en la matière (sur ce point, voir B. Bru, 1988). Mais l'influence qu'il a pu exercer sur Condorcet est, ici, sans nul doute marginale. En effet, d'Alembert est à proprement parler un théoricien des mathématiques mixtes qui, à ce titre, n'a cessé de s'interroger sur les applications de l'analyse mathématique aux autres domaines de la connaissance, ce qui n'est pas le cas de Turgot. De plus, Condorcet se penche sur cette question dès la fin des années $1760^{29}$, alors même qu'il ne connaît pas encore Turgot personnellement et qu'il est en revanche lié à d'Alembert depuis le début de la décennie.

C'est donc avant tout sous l'influence de d'Alembert que Condorcet rejette tout traitement hypothétique des facteurs moraux, du fait de l'incapacité à représenter, de façon uniforme, les idiosyncrasies des individus. Au début des années 1770, Condorcet refuse ainsi d'endosser le principe de l'espérance morale posé par D. Bernoulli - principe sur lequel, du reste, Turgot ne s'exprime jamais explicitement. En effet, il convient «d'écarter toutes les considérations tirées de l'état des joueurs », c'est-à-dire de leur situation pécuniaire, car « il serait impossible, si on y avait égard, de porter aucun jugement fixe » (Faire entrer dans le calcul l'effet des événements, MS 865, f. 85 ; BC, p. 299).

${ }^{29}$ Voir Arithmétique politique (1768), MS 874, f. 297 ; BC, p. 228. 
A la même époque, Condorcet réprouve d'ailleurs la représentation abstraite du processus de marché et, partant, des comportements d'offre et de demande, que l'on trouve dans les Méditations sur l'économie politique de P. Verri, dont la première édition parait en 1771 : «l'envie d'acheter et celle de vendre ne sont susceptibles d'aucun calcul» (Lettre de Condorcet à Verri, 7 nov. 1771 ; BC, p. 73). De surcroît, cette envie s'exprime de façon versatile, elle se trouve dirigée par «l'opinion et les passions » et l'image que Condorcet donne du commerçant est celle d'un individu «borné » (Lettre de Condorcet à Verri, début 1772, BC, p. 73 $)^{30}$. Aussi, les préférences que les agents accordent aux marchandises et le désir qu'ils ont de vendre certaines d'entre elles afin d'acquérir une somme monétaire ne peuvent-ils recevoir, d'après Condorcet, une mesure mathématique abstraite.

Reste maintenant à savoir si ce refus d'une formalisation hypothétique des comportements moraux sera maintenue par Condorcet. La représentation qu'il se donne de l'action de voter, telle qu'on la trouve dans ses textes de maturité, constitue un premier indice incitant à répondre par l'affirmative : se trouve privilégiée ce que nous nommerions aujourd'hui une optique non pas cardinale mais précisément ordinale. Aux yeux de Condorcet, le votant déclare ainsi sa préférence pour un candidat sur un autre, mais ne manifeste en aucun cas le degré d'estime respectif qu'il leur porte : «lorsqu'un électeur est consulté pour savoir si un tel homme proposé est digne ou indigne de la place, on ne doit pas supposer qu'il prononce sur cette question, prise dans un sens absolu : il la considère nécessairement dans un sens relatif» (Sur la forme des élections, 1789, OC, t. IX, p. 297) 31 . G. T. Guilbaud (1952) affirme à ce sujet que Condorcet «a partout cherché à raisonner dans l'ordinal ». De même, K. J. Arrow (1973) estime que la méthode de Condorcet ${ }^{32}$ « is in the fullest ordinalist spirit $\gg$.

En outre, le jugement que Condorcet exprime à l'encontre de la formule bernoullienne, au milieu des années 1780, confirme la continuité de son opinion. Certes, dans

\footnotetext{
$30 \mathrm{Au}$ demeurant, une telle vision semble s'étendre à tous les agents économiques d'après Condorcet - à l'exception, nous le verrons, des « hommes sages ».

31 Ou encore : « celui qui vote en faveur d'un des candidats, prononce bien qu'il le croit supérieur à chacun des autres, mais ne prononce point son opinion sur leur mérite respectif », Essai sur les assemblées provinciales (1788), OC, t. VIII, p. 194.

32 Ce que K. J. Arrow nomme la «méthode» de Condorcet consiste à remplacer le scrutin majoritaire traditionnel par un mode d'élection prenant en compte les ordres de préférence individuels et ce, grâce à une série de comparaisons par paires de candidats. Si cette procédure mène à des résultats contradictoires, c'est-àdire si le «paradoxe des votes » - supposé ici connu du lecteur - apparaît, il conviendra alors, selon Condorcet, d'éliminer la comparaison dont le support de voix est le plus faible. Sur ce point, se reporter au Discours préliminaire de l'Essai (1785), OB, p. 61 et suiv.
} 
ses Notes sur la Thèse de Nicolas Bernoulli (1785-86), il reconnaît que le cousin de ce dernier a fait remarquer « avec raison que la valeur d'une même somme d'argent n'est pas égale dans toutes les circonstances ni pour tous les hommes. Que cent livres par exemple ont une plus grande valeur pour un homme qui a mille livres de bien que pour celui [qui] en a cent mille » (MS 875, f. 181 ; BC, p. 582). Mais, précise-t-il un peu plus loin, « ce principe est hypothétique en lui-même » et, ajoute-t-il enfin, « il nous paraît arbitraire de prendre soit la loi proposée par M. Daniel Bernoulli, soit une autre loi qui remplirait les mêmes conditions » (MS 875, f. 182 ; BC, p. 584) : la critique d'alembertienne de l'espérance morale est bel est bien réitérée par Condorcet - et on a tout lieu de penser que l'encyclopédiste refuse là encore, plus largement, l'idée même de donner une mesure hypothétique aux comportements moraux, associés à quelque objet que ce soit.

Pour autant, tout en refusant l'expression morale de l'espérance, Condorcet rejette-t-il également son estimation mathématique ? C'est ce qui mérite à présent d'être examiné.

\section{3) La réhabilitation de la règle de l'espérance mathématique dans les jeux de hasard}

On estime habituellement que Jacques Bernoulli, oncle de Daniel et de Nicolas, est le fondateur du premier théorème de l'inférence statistique, considéré aujourd'hui comme la «version faible» de la loi des grands nombres. Dans la quatrième partie de son ouvrage posthume, l'Ars Conjectandi (1713), il établit ainsi que lorsque le nombre des épreuves augmente, la probabilité que la fréquence s'écarte de la probabilité de l'événement est de plus en plus petite 33 .

Comme L. J. Daston (1988, p. 98) l'a relevé, l'originalité de Condorcet va être de s'inspirer du théorème de $\mathrm{J}$. Bernoulli afin de résoudre les objections de d'Alembert vis-à-vis de la règle de l'espérance mathématique dans les jeux de hasard. Si les premiers travaux qu'il réalise à cette fin datent du début des années $1770^{34}$, il faudra cependant attendre la publication de la «Première partie » (1784) de son Mémoire sur le calcul des probabilités pour que son argumentation gagne sa pleine maturité. Dans ce texte, Condorcet commence

33 Jacques Bernoulli avait déjà effectué une démonstration algébrique de son théorème, vers 1689-90, à l'article 151a de son journal scientifique, les Meditationes. Pour un commentaire, voir notamment N. Meusnier (1987), (1989).

34 Voir Première ébauche d'essai sur les probabilités (1770), MS 883, f. 221, Z 30, f. 1-3 ; BC, p. 253-255 ; Détermination de la mise d'un joueur (vers 1770), MS 875, f. 110-112 ; BC, p. 274-276 ; et Application des principes aux jeux de hasard et aux événements de la vie (vers 1770), MS 875, f. 116-121 ; BC, p. 264-270. 
par examiner la notion de «jeu égal» - c'est-à-dire de «jeu équitable», selon une terminologie moderne. Il prend à cet égard le cas de deux individus se lançant dans un jeu où il n'existe que deux événements possibles, dont les probabilités sont $e$ et $e$. L'événement ayant pour probabilité $e$ fait gagner la somme $a$ au premier joueur et perdre la même somme au second, l'événement dont la probabilité est $e$ ' fait gagner la somme $a$ ' au second joueur et perdre la même somme au premier. Condorcet explique que le jeu est «égal» si les espérances de gain des deux joueurs coïncident, c'est-à-dire si e'a'=ea (BC, p. 388). Il ajoute un peu plus loin qu'à mesure que le jeu est répété, chacun des joueurs a une plus grande probabilité de gagner effectivement une somme totale correspondant à l'espérance de son gain. Plus précisément, le jeu doit être ainsi renouvelé un nombre suffisamment grand de fois de sorte que :

$1^{\circ}$ le cas le plus probable est justement celui où la situation pécuniaire des joueurs n'est pas changée;

$2^{\circ}$ les probabilités de gagner ou de perdre pour les deux joueurs convergent vers $\frac{1}{2}$;

$3^{\circ}$ il existe une probabilité toujours croissante que la perte subie par chacun des deux joueurs n'excédera pas un montant donné ou, du moins, une partie proportionnelle de la mise totale 35 .

35 De façon formelle, si l'on désigne par $G_{n}$ et $M_{n}$ le gain et la mise, par $\left(G_{n}-M_{n}\right)$ le gain net à chaque partie n, et par $N$ un nombre élevé de parties jouées, ces trois conditions peuvent s'énoncer comme suit :

$1^{\circ}$ La probabilité $\operatorname{Pr}\left(\sum_{n=1}^{N}\left(G_{n}-M_{n}\right)=0\right)$ est maximale ;

$2^{\circ} \quad$ La probabilité $\operatorname{Pr}\left(\sum_{n=1}^{N}\left(G_{n}-M_{n}\right)>0\right)$ est asymptotiquement égale à la probabilité $\operatorname{Pr}\left(\sum_{n=1}^{N}\left(G_{n}-M_{n}\right)<0\right)$;

$3^{\circ} \alpha$ désignant une somme fixe et $P_{n}^{\alpha}$ la probabilité que le gain net total au coup $n$ soit supérieur à $\alpha$, alors la suite $P_{n}^{\alpha}=\operatorname{Pr}\left(\sum_{p=1}^{n}\left(G_{p}-M_{p}\right)<\alpha\right)$ est croissante. Ou du moins : $M$ désignant une partie proportionnelle de la mise totale $\left(M=x \sum_{n=1}^{N} M_{n}\right.$ avec $\left.x \in\right] 0,1\left[\right.$ ) et $P_{n}^{M}$ la probabilité que le gain net total au coup $n$ soit supérieur à $M$, alors la suite $P_{n}^{M}=\operatorname{Pr}\left(\sum_{p=1}^{n}\left(G_{p}-M_{p}\right)<M\right)$ est croissante. Voir Mémoire sur le calcul des probabilités (1784), BC, p. 390 ; Probabilité (1785) ; BC, p. 501-502 ; Discours préliminaire de l'Essai (1785), OB, p. 75-76 ; Notes sur la thèse de N. Bernoulli (1785-86), MS 875, f. 183 ; BC, p. 579. 
Si tel est le cas, l'espérance mathématique peut dès lors servir de guide pour l'action. Dans la même optique, Condorcet entend également par « jeu égal» le fait qu’un ou plusieurs joueurs misent une somme correspondant à l'espérance mathématique de leur gain 36 , à partir du moment où il sont assurés de pouvoir jouer un grand nombre de coups.

Par là même, les doutes que d'Alembert énonce à l'encontre de la règle de l'espérance se trouvent incontestablement fragilisés. Condorcet en a bien évidemment conscience. Ainsi, dans l'un de ses premiers manuscrits probabilistes, alors même qu'il a à peine ébauché son interprétation particulière de l'espérance, que l'on pourrait qualifier de statistique, il l'envisage déjà comme «une réponse aux objections qui ont été proposées contre elle» (Détermination de la mise d'un joueur, vers 1770, MS 875, f. 111 ; BC, p. 276) 37 . Prenons un cas numérique parmi ceux que d'Alembert affectionne le plus, à savoir celui où il s'agit de comparer des espérances équivalentes dont les probabilités respectives sont éloignées les unes des autres. Par exemple, une probabilité $\frac{1}{100}$ de gagner 99000 écus serait délaissée, selon d'Alembert, au profit d'une probabilité $\frac{99}{100}$ d'en gagner 1000. En ce sens, si un joueur était confronté à la première de ces probabilités de gain, il ne s'engagerait pas, par prudence, à jouer avec un autre joueur ayant la seconde de ces probabilités. A cela Condorcet répondrait que si le nombre de coups joués est élevé, le premier joueur perdrait certes souvent mais finirait par gagner effectivement 99000 écus. Sur le long terme, son « état », c'est-à-dire sa situation pécuniaire, demeurerait ainsi inchangé. Autrement dit, « dans tous les cas où les probabilités de gagner ou de perdre sont très différentes entre elles, il faut supposer un grand nombre de coups pour que cette égalité [entre l'état avant le jeu et celui après le jeu] ait lieu sensiblement » (Eléments, 1786-87, OB, p. 559).

Malgré les amendements que Condorcet tente d'apporter à la règle de l'espérance mathématique, sa capacité à guider la décision ne va cependant pas de soi. Ainsi, elle ne peut s'appliquer au problème de Petersbourg puisque, comme l'on ne sait pas quand C adviendra, le nombre de coups et la mise établis d'après la règle de l'espérance mathématique sont infinis - il faudrait alors recommencer la partie un nombre infini de fois pour espérer compenser gains et pertes (Détermination de la mise d'un joueur, vers 1770, MS 875, f. 111 ;

\footnotetext{
36 Voir l'article Probabilité (1785), BC, p. 501. Se reporter également à la troisième des Notes sur la thèse de Nicolas Bernoulli (1785-86), MS 875, f. 183-184 ; BC, p. 580-581; MS 875, f. 181 ; BC, p. 583.

37 B. Bru (1994) considère d'ailleurs que Condorcet est le fondateur de la "première véritable théorie de l'espérance », de surcroît définie, au début de la « Première partie » (1784) de son Mémoire sur le calcul des
} 
BC, p. 276) ${ }^{38}$. Par ailleurs, dans son analyse des loteries (Eléments, 1786-87, OB, p. 560579), Condorcet explique que le banquier n'est pas certain de pouvoir rejouer un grand nombre de coups puisqu'il risque la ruine à court terme. Afin de détenir une très grande probabilité de ne pas faire banqueroute au bout d'un petit nombre d'épreuves, il va alors exiger une espérance de gain supérieure à son enjeu en réclamant un bénéfice plus grand. Les pontes, quant à eux, acceptent de s'engager à ce « jeu inégal » puisque leur mise très faible, ne mettant pas en péril leur fortune personnelle, les entretient dans l'espoir de gagner une somme considérable 39 .

La situation dans laquelle se trouve le banquier met en exergue la contradiction pouvant survenir entre la règle de l'espérance mathématique et l'impératif de prudence, contradiction déjà exprimée par d'Alembert. En l'occurrence, le banquier ne peut établir ses spéculations à partir du principe de l'espérance que s'il envisage simultanément un seuil à partir duquel le risque de ruine peut être considéré comme négligeable ; l'intégration effective de ce seuil se traduisant finalement par la perception d'une prime de risque d'où résulte une espérance de gain supérieure à sa mise.

Rappelons cependant que d'Alembert fait preuve d'un scepticisme indiscutable lorsqu'il exprime la nécessaire négligence que les individus, par prudence, doivent affecter à l'encontre d'un risque : quelle valeur assigner aux probabilités juste supérieures à ce risque, doivent-elles être considérées comme plus faibles que leur valeur mathématique et dans l'affirmative, comment déterminer et jusqu'à quel terme appliquer la loi de diminution de la probabilité qui s'appliquerait à leur égard ? Si Condorcet examine lui aussi le problème de l'établissement d'un seuil de probabilité, il ne s'embarrasse pas pour autant de telles interrogations. En ce sens, et à la différence de d'Alembert, il tente d'attribuer une mesure précise à l'impératif de prudence.

probabilités, pour plusieurs événements, certains d'entre eux amenant un avantage positif, d'autres un avantage négatif. Sur ce second point, voir B. Bru \& P. Crépel (1994), p. 387.

38 Condorcet expose à plusieurs autres reprises dans son œuvre l'inapplicabilité de la règle de l'espérance mathématique au problème de Petersbourg. Voir par exemple Première ébauche d'essai sur les probabilités (1770), MS Z 30, f. 2 ; BC, p. 255 ; « Première partie » (1784) du Mémoire sur le calcul des probabilités, BC, p. 392.

39 On remarque que cette idée est également formulée par Smith : «que l'on surestime naturellement les chances de gain, c'est ce qui ressort du succès universel des loteries. On n'a jamais vu, et l'on ne verra jamais, de loterie parfaitement équitable [...] l'espérance vaine de gagner quelques-uns des gros lots est l'unique cause de cette demande » - la demande en question est celle de billets de loteries organisées par l'Etat, billets dont le prix dépasse la valeur de l'espérance mathématique du gain qu'ils sont susceptibles de procurer ; Smith (1776), vol. I, p. 125. 
Dans cette perspective, lorsqu'il envisage les spéculations économiques de l'entrepreneur, Condorcet combine la règle de l'espérance à une série de seuils de probabilités, chacun relatif à un risque considéré comme plus ou moins grave - dont celui de ruine. Ce faisant, il élabore une véritable théorie du choix économique en univers risqué, sur laquelle nous allons maintenant lever le voile.

\section{La théorie condorcétienne du comportement économique en univers risqué}

L'analyse relativement approfondie du risque économique effectuée par Condorcet peut d'abord être illustrée par son examen du négoce maritime. Afin de décrire le comportement du négociant et de l'assureur, Condorcet adjoint ainsi à la règle de l'espérance mathématique une série de seuils probabilistes, plus ou moins élevés selon l'ampleur du danger envisagé (3. 1). Mais la réflexion de l'académicien s'étend également à n'importe quel type d'entreprise de commerce et, plus largement encore, aux entreprises de culture et d'industrie, quel que soit le montant des capitaux engagés (3. 2). Par la même occasion, Condorcet expose ce qu'il estime devoir être la rémunération de l'entrepreneur. Il conviendra alors de comparer ses conceptions à celles de Smith et de Turgot, ces deux écrivains étant habituellement considérés comme les fondateurs de la théorie classique du profit 40 . Sous deux points de vue essentiels, ce revenu apparaît en effet chez eux comme une catégorie spécifique, ce qui n'est pas le cas dans le discours de leurs devanciers : d'une part, le profit dépend de la grandeur des valeurs accumulées par une classe particulière, celle des entrepreneurs capitalistes ; d'autre part, il est distingué du salaire - l'analyse de Turgot étant, sur ce second point, toutefois plus fragile que celle de Smith. Or, si Condorcet semble s'inspirer, à certains égards, de ses deux prédécesseurs, ce sont néanmoins les réflexions de d'Alembert sur la formalisation de l'aléatoire qui le mènent à prendre très scrupuleusement en compte le risque économique, ce qui donne du même coup à sa théorie du profit une tournure incontestablement originale (3.3). Ces diverses réflexions, concernant le commerce maritime et plus largement n'importe quel type d'entreprise économique, rouleront cependant sur une hypothèse : les probabilités de perte et de gain ainsi que les différents seuils de probabilité qui

40 Sur la théorie du profit de Turgot, voir, entre autres références, J. T. Ravix \& P. M. Romani (1982) et G. Faccarello (1992). Concernant celle de Smith, nous renvoyons à la contribution, ancienne mais particulièrement fine, de R. L. Meek (1954). 
interviennent dans l'analyse sont supposés connus. Au terme de notre étude, il nous faudra lever cette hypothèse. En cela, nous ne faisons que calquer la progression du raisonnement de Condorcet lui-même 41 . C'est alors le caractère empirique de la théorie condorcétienne du comportement économique en univers incertain que nous tâcherons de mettre en évidence (3.4). Nombreux sont en effet les passages où Condorcet insiste sur la nécessité de recueillir un certain nombre de données concrètes pour établir son analyse, comme s'il cherchait à rappeler que la conformité de l'art de conjecturer aux phénomènes réels, revendiquée par d'Alembert, se trouve simultanément satisfaite. Aussi, de tels passages semblent-ils constituer autant d'échos aux préoccupations de son maître.

\section{1) Le commerce maritime}

Liées au développement du grand commerce à la fin du Moyen-Age puis au moment de la Renaissance, les assurances maritimes connurent toutefois une conceptualisation relativement tardive. A peine ébauchée par Jacques Bernoulli dans les articles 77 b et 87 de ses Meditationes (1677-1705) et par G. J. 's Gravesande à l'occasion de son Introduction à la philosophie (1736, p. 134 \& 144), l'approche théorique de ce mode d'assurance, pourtant beaucoup plus répandu que celui sur la vie ou les incendies, n'est véritablement développée que par Nicolas Bernoulli, dans L'usage de l'art de conjecturer en droit (1709, p. 94-98). Avant que Condorcet ne se penche sur ce problème, la seconde publication notable en la matière est l'Esquisse d'une théorie nouvelle de mesure du sort (1738) de Daniel Bernoulli, texte à l'occasion duquel, nous l'avons évoqué, ce troisième membre de l'illustre lignée des mathématiciens bâlois défend une interprétation psychologique, morale, de l'espérance.

Condorcet fait en revanche intervenir la règle de l'espérance mathématique afin de rendre compte du comportement de l'assureur, comme Nicolas Bernoulli. Cependant, son analyse est non seulement plus riche mais également plus complexe que celle de ce dernier, puisqu'il étudie aussi le comportement du négociant et qu'il mêle de surcroît à la règle de

\footnotetext{
41 En guise d'exemple, on peut citer un passage de l'article «Assurances maritimes » (1784) : «nous avons supposé jusqu'ici que l'on connaissait, $1^{\circ}$ la probabilité de la perte de chaque bâtiment qu'on se propose d'assurer ; $2^{\circ}$ le degré de probabilité qu'un négociant ou un assureur doit avoir de ne point perdre pour qu'il puisse s'exposer à un risque, sans mériter qu'on l'accuse d'imprudence ». Condorcet ajoute : « il faut donc chercher à connaître ces deux données »; $\mathrm{BC}, \mathrm{p} .491$. Voir également les Précisions sur le programme du prix relatif aux assurances maritimes (vers 1783, BC, p. 468) et les Eléments (1786-87), OB, p. 562.
} 
l'espérance mathématique une série de niveaux de probabilité, plus ou moins élevés selon l'ampleur du risque envisagé.

$\mathrm{Au}$ début de son article «Assurances maritimes» $(1784)^{42}$, paru dans la partie mathématique de l'Encyclopédie Méthodique, Condorcet raisonne à partir d'une contrainte probabiliste uniforme, relativement souple : le négociant doit détenir « une espérance assez grande de retirer, avec ses avances, leur intérêt et le salaire commun de son travail » (BC, p. 487). Afin de ne pas laisser planer une quelconque confusion, il convient de préciser que Condorcet assigne ici une connotation probabiliste au terme d' "espérance ». Comme un passage précédent de son texte le laisse supposer 43 , 1'expression « espérance assez grande » doit donc s'entendre comme une «probabilité assez grande». Pour bien fixer le raisonnement, indiquons également que le salaire du négociant, ses avances mais aussi leur intérêt - sans quoi il supporterait ce que nous appellerions aujourd'hui un «coût d'opportunité » - représentent ici sa mise selon Condorcet. Ainsi, le commerçant ne va pas se contenter d'une espérance de profit correspondant à sa mise. Il lui est de surcroît nécessaire de bénéficier d'une « probabilité assez grande » de récupérer celle-ci, c'est-à-dire d'avoir une probabilité de la couvrir supérieure à $\frac{1}{2}$, même si Condorcet ne le précise pas explicitement. Or, une espérance de profit dépassant le montant de son enjeu lui permettra précisément de satisfaire cette contrainte probabiliste. Le profit qu'il va exiger doit en conséquence s'élever.

Seulement, Condorcet explique que la pression concurrentielle ne permet pas au négociant de percevoir un tel profit. Deux solutions s'offrent alors à lui. La première consiste à répartir davantage ses risques (ibid., p. 488). En multipliant ainsi le nombre de «parties » qu'il joue, le négociant a plus de chances de récupérer sa mise. Sans pour autant envisager l'accroissement des coûts de transport que susciterait cette dispersion accrue des risques, Condorcet note toutefois qu'elle est difficilement applicable dans la pratique, les marchandises du négociant n'étant pas divisibles selon son bon gré. La seconde solution dont il dispose, plus vraisemblable, est de s'assurer. Il pourra, dans ce cas, bénéficier d'un profit plus faible que celui lui garantissant une «probabilité assez grande » de récupérer sa mise. L'assureur, quant à lui, se contentera alors d'un profit inférieur à celui du négociant puisqu'il

\footnotetext{
42 Pour un commentaire de cet article, et notamment une traduction en langage mathématique moderne des divers raisonnements qui y sont effectués, voir P. Crépel (1988 a).

43 Condorcet écrit en effet quelques lignes plus haut : « un homme raisonnable ne doit se livrer au commerce que dans le cas où il trouve une probabilité assez grande [n. i.] qu'il retirera ses fonds, avec l'intérêt commun et le prix de son travail »; BC, p. 486.
} 
peut répartir davantage ses risques - il est ici sous-entendu qu'il contracte avec une multitude de clients. Condorcet conclut : « le taux de l'assurance se détermine donc pour chaque espèce de risque par un certain milieu que la concurrence établit entre la partie du profit que le négociant peut abandonner, et celle qui est nécessaire à l'assureur pour avoir une très grande probabilité de gagner » (ibid.).

Les probabilités de naufrage et d'arrivée à bon port des navires étant supposées connues, il reste à connaître «les deux limites du taux d'assurance », c'est-à-dire la prime d'assurance minimale et maximale que négociant et assureur peuvent respectivement exiger. Ces deux limites doivent être établies de telle sorte qu'elles garantissent aux deux parties un bénéfice non seulement positif, mais encore au moins aussi élevé que celui résultant d'entreprises « où il n'y a pas de risque » (ibid.). En des termes plus modernes, Condorcet cherche ainsi à déterminer les deux « prix de réserve » de l'assurance, entre lesquels le contrat sera considéré comme avantageux par l'assureur et le négociant, tous deux devant par ailleurs éviter de supporter un « coût d'opportunité ». Néanmoins, Condorcet n'indique pas la nature exacte des investissements alternatifs, non risqués, que négociant et assureur pourraient effectuer. Nous reviendrons plus loin sur ce point. Il suffit pour l'instant de constater que son propos est ici relativement imprécis car, d'un point de vue littéral, Condorcet commence par exclure implicitement le salaire dans la rémunération des entreprises « où il n’y a pas de risque $»^{44}$ - ce qui laisserait entendre qu'il s'agit là de placements en bien-fonds - pour ensuite l'intégrer dans sa démonstration mathématique. Les calculs effectués par Condorcet sont établis à partir de la série de notations suivante :

- Somme risquée dans chaque entreprise $=a$

- Nombre d'entreprises sur lesquelles le négociant risque $a=n$

- Taux d'intérêt résultant d'une entreprise non risquée $=c$

- Profit que le négociant doit retirer du « salaire de son temps et de ses peines » $=C^{\text {' }}$

- Probabilité nécessaire pour se lancer dans le commerce avec prudence $=P$

- Nombre de naufrages que le négociant peut tolérer $=m$ (supposé déduit de $P$ )

- Profit résultant de chaque entreprise $=b$

- « Salaire de la peine » éprouvée par l'assureur $=e$

- Nombre de navires garantis par l'assureur $=n$,

44 « Soit $a$ la mise première du négociant $[\ldots] a\left[(1+c)^{2}\right]$ est ce qu'il doit recevoir au bout de deux ans, $c$ étant le denier d'intérêt pour les entreprises où il n'y a pas de risque, soit de plus c' le profit qu'il doit tirer de cette entreprise [risquée] comme salaire de son temps et de ses peines [n. i.]», ibid.. 
- Probabilité, exigée par l'assureur, de ne pas perdre sur les $n$ ' navires $=P$ '

- Nombre de naufrages que l'assureur peut tolérer $=m$ ' (supposé déduit de $P^{\prime}$ )

- Probabilité pour un navire d'arriver à bon port $=g$

- Probabilité pour un navire de faire naufrage $=p(p=1-g)$

- Prime d'assurance versée par le négociant et touchée par l'assureur $=b$ '

Le gain espéré par le négociant s'il plaçait la somme $a$ pendant deux ans dans une entreprise non risquée s'élève à : $a\left[(1+c)^{2}\right](1)$. S'il se lance dans le commerce maritime, le négociant doit récupérer, pour chaque entreprise, ses avances $a$ ajoutées d'un profit $b$, somme devant non seulement couvrir celui résultant d'une entreprise non risquée (1) ainsi que le « salaire de son temps et de ses peines »c', mais également demeurer supérieure à la prime d'assurance $b^{\prime}$ versée à l'assureur. On a donc : $b^{\prime}<b-a\left(2 c+c^{2}+c^{\prime}\right)(2)$. Le deuxième terme de cette inéquation - c'est-à-dire le « surcroît de profit » désiré pour chaque entreprise - multiplié par le nombre minimum de non perte des marchandises exigé par le négociant, $(n-m)$, doit être supérieur au profit qu'il percevrait s'il plaçait son argent dans une entreprise non risquée, multiplié par le nombre tolérable de naufrages $m$. Autrement dit, le surcroît de profit total est proportionnel à la probabilité $P$ nécessaire pour se lancer dans le commerce avec prudence $m$ étant déduit de $P$. Ce qui revient par conséquent à écrire :

$$
(n-m)\left[b-a\left(2 c+c^{2}+c^{\prime}\right)\right]>m \cdot a\left[(1+c)^{2}+c^{\prime}\right](3)
$$

L'assureur quant à lui doit bénéficier d'une prime d'assurance totale $n$ 'b' supérieure à la somme maximale qu'il peut verser au négociant, ajoutée de son salaire. Pour ce qui le concerne, on doit donc avoir :

$$
n^{\prime} b^{\prime}>m^{\prime}(a+b)+e \text { soit } b^{\prime}>\frac{m^{\prime}}{n^{\prime}}(a+b)+\frac{e}{n^{\prime}}(4) \text {. }
$$

En supposant que l'inégalité (3) est vérifiée, les propositions (2) et (4) indiquent les prix de réserve respectifs du négociant et de l'assureur. En prenant ces deux propositions comme des égalités, Condorcet en déduit le niveau minimum du profit pour le négociant lorsque le jeu de la concurrence s'exerce, à savoir :

$$
b=\left(\frac{m^{\prime}}{n^{\prime}}+2 c+c^{2}+c^{\prime}\right) \frac{n^{\prime}}{n^{\prime}-m^{\prime}} a+\frac{e}{n^{\prime}-m^{\prime}} \text { (ibid., p. 488-489). }
$$

Après avoir brièvement évoqué les modifications du rapport négociant-assureur lorsque le négociant, ayant déjà envoyé ses marchandises en mer, voit ses risques s'accroître au point d'exposer toute sa fortune du fait d'événements imprévus, Condorcet complexifie davantage son discours en exposant une série de contraintes probabilistes relativement fines : 
« supposons en effet qu'un négociant risque une somme $a$, il sera possible qu'il se contente d'une probabilité $P$ de retirer $a$, d'une probabilité $P$ ' de ne perdre que la partie de son profit, destinée à le dédommager de ses peines, d'une probabilité encore plus grande $P$ '” de ne perdre que les intérêts de ses fonds, et qu'il ne cherche que la certitude de ne pas entamer ses fonds au-delà d'un certain terme » (ibid., p. 490) 45 , sans quoi il risquerait la ruine au bout d'un petit nombre de coups, et ne pourrait alors pas se fonder sur la règle de l'espérance mathématique pour établir ses spéculations. N'étant pas en mesure de répartir ses risques indéfiniment ni exiger un profit supérieur à celui fixé par la concurrence afin de satisfaire de telles contraintes, le négociant va chercher à souscrire divers types d'assurances couvrant une partie plus ou moins grande de ses fonds. L'assureur lui-même fondera ses spéculations en établissant une série de seuils de probabilités qu'il s'agira de ne pas dépasser afin de s'engager, avec profit, dans un contrat avec le commerçant (ibid., p. 490-491). Nous ne détaillerons pas les calculs que Condorcet effectue à cette occasion : au demeurant assez sommaires, ils sont uniquement relatifs aux évaluations du négociant et ne débouchent pas sur la détermination du prix de réserve de l'assureur.

Si le développement mathématique effectué par Condorcet quand il raisonne à partir d'une contrainte probabiliste uniforme marque pour lui l'occasion d'établir, en revanche, les prix de réserve du négociant et de l'assureur, on relève cependant le fait que l'encyclopédiste n'aboutit pas à une détermination formelle de la prime d'assurance fixée à l'issue de la négociation. En ce sens, il n'énonce pas ici une théorie mathématique du prix de marché en tant que tel, ce qui demeure d'ailleurs le cas dans le reste de son œuvre. Son analyse est, sous ce rapport, nettement moins audacieuse que celle développée par Lloyd (1771, chpt IX), Frisi (1772) et, un peu plus tard, Canard (1801, chpt III) et Bicquilley (1804, chpt III). Condorcet s'approche néanmoins d'une telle formulation, mais d'une façon littérale, lorsqu'il explique que la prime d'assurance est déterminée «par un certain milieu », établi par la concurrence, entre les exigences du négociant et de l'assureur. Il parle en outre, deux lignes plus bas, d'un «prix moyen» (ibid., p. 488) 46 . Pourquoi ne franchit-il pas le pas ? Parce que son raisonnement serait alors tombé dans un cadre hypothétique ? Il est difficile de le savoir

\footnotetext{
45 La part du profit nécessaire pour dédommager le négociant « de ses peines » correspond à son salaire.

46 Le terme de « prix moyen » employé ici par Condorcet n'a pas la même signification que dans certains autres de ses textes où, s'inspirant de Quesnay (1757, p. 462 et suiv.), il entend par ce terme la moyenne arithmétique du prix pondérée par les quantités produites, sur plusieurs périodes.
} 
même si, sur ce point comme sur d'autres, il convient de garder à l'esprit l'emprise intellectuelle de d'Alembert sur son disciple...

Quoi qu'il en soit, il s'agit maintenant de montrer que l'argumentation employée par Condorcet, fondée sur une interprétation statistique de l'espérance mathématique et une batterie de contraintes probabilistes en fonction de la gravité du risque encouru, doit également s'appliquer selon lui à n'importe quel type d'entreprise économique, excepté l'affermage de terre, ce dernier placement servant, en quelque sorte, de référent.

\section{2) Entreprise économique, entreprise risquée}

Dans ses Eléments (1786-87), Condorcet examine les spéculations d'un négociant dans une entreprise non spécifiquement maritime, lorsque le risque auquel il se trouve confronté est dit « sensible» (OB, p. 561). Là encore, affirme Condorcet, le commerçant ne va pas fonder ses spéculations sur une espérance mathématique de profit qui, au bout d'un grand nombre d'épreuves, lui permettrait de récupérer les différentes composantes de sa mise et de faire, pour ainsi dire, «jeu égal» avec le hasard des événements. Il peut en effet être menacé de ruine avant même d'avoir répété son entreprise un nombre suffisamment grand de fois. En outre, il s'agit pour lui d'avoir de grandes chances de bénéficier d'un profit positif. De là, il va établir plusieurs seuils de probabilité, chacun relatif à un risque considéré comme plus ou moins grave. D'où l'exigence de voir s'accroître l'espérance mathématique de son profit, ce qui lui permettrait justement d'être quasiment certain de ne pas être ruiné et de récupérer tout ou partie de sa mise au bout d'un nombre limité de coups et d'avoir, a fortiori, une probabilité chaque fois plus grande de gagner un certain profit à mesure que le nombre de coups joués augmente. Pour ce faire, le négociant va exiger, selon les propres termes de Condorcet, un «excès de profit» qui lui garantit une espérance mathématique de profit suffisamment grande afin de satisfaire de telles contraintes. Aussi Condorcet reproduit-il exactement le même type de raisonnement que lorsqu'il examine le commerce maritime :

« dans les circonstances où un négociant fait une spéculation accompagnée d'un risque sensible, on voit qu'il ne suffirait point que son profit fût tel que la valeur moyenne de ses espérances fût égale à sa mise, augmentée de l'intérêt qu'un commerce sans risque lui aurait procuré. Il faut encore qu'il ait, en continuant ce genre de commerce, une très grande probabilité qu'il n'essuiera à la longue aucune perte. Il faudrait donc, pour soumettre au calcul les spéculations de ce genre, déterminer, d'après les fonds que chaque commerçant peut avoir à employer successivement dans ce commerce périlleux, quel excès de profit il faut qu'il trouve pour avoir une probabilité suffisante de ne point perdre la totalité de ses fonds, de ne les perdre qu'en partie, de les conserver seulement, de les conserver, plus un certain intérêt » (ibid., p. 561-562). 
Cette longue citation contient cependant certaines ambiguïtés qu'il convient de dissiper.

Tout d'abord, comme nous l'avons déjà fait remarquer, le vocabulaire dont Condorcet use afin de désigner la règle de l'espérance mathématique est assez fluctuant. Ainsi, par « valeur moyenne de ses espérances », on doit entendre ici l'espérance mathématique du gain du négociant.

D'autre part, le statut de l'expression de «commerce sans risque » que Condorcet utilise n'est pas bien déterminé. En effet, il commence par invoquer la nécessité de fixer des seuils de probabilité lorsque l'on effectue dans le commerce une «spéculation accompagnée d'un risque sensible». Dans ce cas, l'expression de "commerce sans risque », dont Condorcet use juste après, devrait en réalité s'entendre comme une opération où le risque peut être négligé, du reste radicalement opposée au «commerce périlleux » dont le marquis parle quelques lignes plus bas. Il ne s'agit pas là du «commerce d'argent» car ce genre de commerce comporte un risque non «négligeable»: celui de la non solvabilité de l'emprunteur. D'ailleurs, lorsqu'il traite des déterminants du niveau du taux de l'intérêt de l'argent dans son Essai sur les assemblées provinciales (1788), Condorcet indique que « la probabilité plus ou moins forte de perdre son capital, ou une partie de son capital, fait exiger des intérêts plus ou moins au-dessus de ce qu'ils auraient été dans un placement assuré » (OC, t. VIII, p. 526). C'est pourquoi, compte tenu du caractère relativement élastique donné au terme de commerce au XVIII ${ }^{\mathrm{e}}$ siècle et d'après la conception couramment admise à cette époque, on serait finalement en droit de penser que Condorcet désigne par l'expression de « commerce sans risque " l'acquisition de bien-fonds. L' « intérêt d'un commerce sans risque », d'un « placement assuré », devrait ainsi s'entendre comme la rémunération plancher résultant d'un affermage de terre i.e d'une opération économique où le risque peut être considéré comme négligeable : le taux de rente. Et, même si nous n'avons pas trouvé, dans l'œuvre de Condorcet, une définition explicite de ce qu'il entend par «commerce sans risque », ce statut de référent accordé à l'intérêt d'un placement en bien-fonds est en outre étayé par l'affirmation selon laquelle «c'est sur l'intérêt de l'argent placé en terres que se règlent tous les autres intérêts » (ibib., p. 284).

Dans son calcul probabiliste afférent aux assurances maritimes, nous avons cependant constaté que Condorcet évalue le profit résultant des entreprises « où il n'y a pas de risque » 
en comptabilisant l'intérêt de celles-ci et le salaire « du temps » et « des peines » $(\mathrm{BC}, \mathrm{p}$. 488), rémunération qui n'aurait pas lieu d'être si le "commerce sans risque » désignait l'acquisition de bien-fonds. Son propos est toutefois assez ambigu puisque nous avons indiqué que, d'un point de vue littéral, le salaire est exclu de ces entreprises - il en est de même dans l'étude du commerce maritime effectuée par S. F. Lacroix (1822, p. 236), le principal disciple de Condorcet. Aussi, afin de maintenir une certaine cohérence dans l'analyse de l'encyclopédiste, nous avons finalement choisi de considérer que l'expression de « commerce sans risque » désigne bel et bien l'affermage de terre.

$\mathrm{Au}$ demeurant, l'assimilation du « commerce sans risque » au placement foncier est confirmée par le fait que Condorcet définit en d'autres endroits le commerce en tant que tel comme une activité intrinsèquement risquée. Au début d'un manuscrit consacré aux assurances maritimes, il effectue ainsi la distinction entre les risques émanant du jeu, en principe négligeables, et ceux encourus dans une opération commerciale, où il demeure en revanche impossible de faire abstraction du risque : « les risques du commerce ne doivent pas se calculer comme ceux du jeu. Quels que soient les principes secrets de la passion du jeu, il ne peut être regardé par un homme raisonnable que comme un divertissement où il ne doit risquer que ce qu'il peut perdre sans exposer son honneur, sa fortune ou celle de sa famille. Le commerce au contraire est un état de toute la vie dans lequel on se propose d'assurer sa subsistance ou d'augmenter sa fortune » (Précisions sur le programme du prix relatif aux assurances maritimes, vers 1783, MS 857, f. 202 ; BC, p. 467). Par conséquent, l'interprétation de la règle de l'espérance comme une moyenne valable lorsque le nombre de coups joués est élevé et la prise en compte de divers niveaux de probabilité en fonction de la gravité des risques auxquels on s'expose s'appliquent ici, non seulement au commerce maritime ou au négoce accompagné d'un risque « sensible», mais également au commerce en général. En d'autres termes,

« un homme raisonnable ne doit se livrer au commerce que dans le cas où il trouve une probabilité assez grande qu'il retirera ses fonds, avec l'intérêt commun et le prix de son travail. Il lui faudrait sans doute une probabilité à peine différente de la certitude de ne pas perdre la totalité de ses fonds, et même de conserver la partie qui est nécessaire à sa subsistance et à celle de sa famille ; et une probabilité encore très grande de ne pas les diminuer jusqu'à un certain point » (Assurances maritimes, 1784, BC, p. 486).

Toutefois, Condorcet ne précise pas en toutes lettres, ici, que la prise en compte de ces différents niveaux de probabilité se réalise par un accroissement de l'espérance du profit. Mais on a tout lieu de penser qu'il conserve à l'esprit ce raisonnement qu'il expose si souvent 
en d'autres endroits. D'ailleurs, et de façon plus large encore, il étend cette même analyse à n'importe quel type d'entreprise économique. Extrait du Mémoire sur le calcul des probabilités (1784), le passage suivant est à ce titre exemplaire :

«[...] dans les entreprises où les hommes s'exposent à une perte, dans la vue d'un profit, il faut que le profit soit plus grand que celui qui suivant la règle générale, établit l'égalité : en effet, comme en général on ne s'y livre point comme au jeu, par l'attrait du plaisir de jouer, ou comme aux loteries, par l'espérance de gagner beaucoup avec une petite mise, on ne peut avoir de motif de risquer, qu'un avantage qui, en envisageant une suite de risques semblables, produise une assurance assez grande de gagner, et une probabilité presqu'égale à la certitude de ne point perdre au-delà d'une certaine partie de la mise » (BC, p. 396-97).

A l'exception de l'affermage de terre, «commerce sans risque » utilisé comme référent, n'importe quel type d'entreprise économique doit donc être guidé, selon Condorcet, par la règle de l'espérance mathématique assortie d'une série de conditions prudentielles dont la prise en compte mène finalement à une élévation de cette espérance. Ainsi, les divers seuils de probabilité établis par l'entrepreneur se concrétisent finalement en une variété de primes de risque qui viennent s'ajouter à son salaire, ses avances et leur intérêt.

Le raisonnement de Condorcet pourrait être symbolisé de la façon suivante :

- $p$ et $g$ désignent les probabilités de perte et de gain de l'entrepreneur.

- $q^{*}$ est le gain désiré par l'entrepreneur. Il intègre les diverses primes de risque qu'il exige.

- $q$ et $m$ représentent son gain et sa mise - c'est-à-dire ses avances, leur intérêt, et le prix de son travail.

- $X_{1}$ est la variable aléatoire correspondant à son profit total après la première entreprise.

L'espérance mathématique $E\left(X_{1}\right)$ de cette variable aléatoire est égale à gq-pm. Si l'on répète $n$ fois l'entreprise, il est naturel de considérer la variable aléatoire $G_{n}=X_{1}+X_{2}+\cdots+X_{n}$ qui représente le profit cumulé, c'est-à-dire total, résultant des $n$ premières entreprises. Condorcet concentre son analyse sur les fluctuations de la variable $G_{n}$ et notamment ses passages par certains niveaux : seuil de ruine, seuils correspondant à la récupération des avances, d'une partie de celles-ci, de leur intérêt et, enfin, niveaux garantissant à l'entrepreneur la couverture du prix de son travail et du bénéfice qu'il est en droit d'exiger pour que son opération s'avère profitable. La prise en compte effective de ces différents seuils le mène à exiger un gain $q^{*}$ supérieur à $q$. Son espérance de profit total souhaitée sera donc supérieure à celle de $G_{n}$. Cette espérance de profit total souhaitée lui permettrait ainsi d'avoir une plus grande probabilité de ne pas être ruiné au terme d'un petit 
nombre d'opérations, de récupérer une partie de ses avances, de bénéficier d'un certain profit etc.

Sous certains aspects, on serait incité à voir en Condorcet le précurseur indûment négligé de contributions plus récentes cherchant en particulier à traiter, dans le cadre de processus stochastiques en temps discret ou continu, du comportement face au risque de ruine qui émanerait, soit de compagnies d'assurance 47 , soit de manière plus générale de n'importe quel type d'entreprise économique 48 . Cependant, il est moins important de signaler un héritage effectif, bien qu'aucun auteur contemporain ne le revendique, que de mentionner ce qui, par-delà les raffinements de l'analyse, singularise la perspective ouverte par Condorcet. C'est à juste titre que P. Crépel (1988 a), lorsqu'il compare les travaux de l'encyclopédiste sur les assurances maritimes avec ceux de certains actuaires contemporains, met en valeur une particularité essentielle du raisonnement de Condorcet, à savoir l'adoption d'un « double point de vue », celui de l'assureur et de l'assuré. D'emblée, l'unité élémentaire de l'analyse réside en effet dans la mise en rapport des spéculations de l'assureur et du négociant, tous deux cherchant à se prémunir de certains risques, dont celui de ruine. D'une certaine façon, la négligence d'un tel problème par les recherches actuelles pourrait s'expliquer par le fait que celles-ci se focalisent sur le transfert du risque de ruine réalisé par l'assureur lui-même : la considération de ce risque mène ce dernier, on le sait, à se réassurer auprès d'autres compagnies ou à constituer des pools avec d'autres assureurs. Aussi, l'optique d'une négociation strictement bilatérale entre assureur et assuré se trouverait-elle, de ce fait même, naturellement occultée. Sous un angle analytique, il n'empêche que Condorcet trace les contours d'un problème qui reste, aujourd'hui encore, à résoudre : celui du double risque de ruine dans le cadre d'une relation d'assurance bilatérale 49 .

Ce qui se dessine ici est l'effet d'une intimité contradictoire entre Condorcet et d'Alembert. Les divergences de vue entre les deux hommes portent sur la valeur scientifique du calcul des probabilités, mais l'un et l'autre se retrouvent, peu ou prou, dans une conception mixte des mathématiques. Or, si cette conception portait en germe une extension possible au

47 Citons par exemple les travaux de B. de Finetti (1940), J. Dubourdieu (1952, chpt V \& VI), H. Cramér (1955), R. E. Beard, T. Pentikaïnen \& T. Pesonen (1969, p. 227-233, 308-318 \& 357-59), H. Bülhmann (1970, chpt VI).

48 A savoir, entre autres études, P. Massé (1953), W. Feller (1966, p. 182-184, 198-199, 326, 377-378, 411 \& 469-470), M. Allais (1953), (1979 a), K. Borch (1967), (1968). 
domaine des sciences politiques, elle était entravée par l'intransigeance de d'Alembert. En mettant cette dernière en cause, Condorcet ouvre la voie à une investigation économique dont le traitement du risque à travers le profit constitue une des manifestations. Afin de mettre en évidence l'originalité de cette contribution de Condorcet, c'est à la théorie du profit de Turgot et de Smith qu'il s'agit maintenant de la comparer.

\section{3) Risque et profit}

Certes, la description de la rétribution de l'entrepreneur livrée par Turgot dans ses Réflexions sur la formation et la distribution des richesses (1766) offre certaines caractéristiques dont Condorcet s'est sans doute inspiré. On décèle ainsi une identité morphologique dans leur définition respective du profit : ce revenu est envisagé comme une somme constituée de plusieurs rétributions (concernant Turgot, voir les Réflexions, t. II, p. 569 \& 591). Cependant, ce type de représentation semble systématique chez Condorcet, ce qui n'est pas le cas chez Turgot, ce dernier établissant parfois une liaison univoque entre le montant du profit et la grandeur des valeurs accumulées (ibid., p. 570 \& 574). Par conséquent, il convient de reconnaître que, sur ce point, le propos de Condorcet se montre paradoxalement moins moderne que celui de son prédécesseur. De façon plus large, et à supposer même qu'il soit fait abstraction du jeu des influences, Condorcet ne développe à aucun endroit de son œuvre une représentation aussi poussée d'une économie fondée sur la reproduction du capital $^{50}$, telle qu'on la trouve dans les Réflexions.

Malgré tout, on remarque que la catégorie du risque n'est pas toujours explicitement intégrée dans son analyse ${ }^{51}$. Quand elle n'est pas mentionnée, il arrive que Turgot y fasse

\footnotetext{
49 A notre connaissance, seul A. Raviv (1979) concentre son étude sur le rapport assureur/assuré. Mais cet auteur n'examine pas pour autant le problème du risque de ruine dont les deux contractants chercheraient à se prémunir.

50 On constate en particulier qu'il n'existe pas chez Condorcet, de réel exposé d'une tendance à «l'uniformisation pondérée des taux de profit», comme la qualifie C. Morilhat (1988), p. 175. Tout au plus peut-on renvoyer à sa remarque allusive, déjà mentionnée, selon laquelle « c'est sur l'intérêt de l'argent placé en terres que se règlent tous les autres intérêts »; Essai sur les assemblées provinciales (1788), OC, t. VIII, p. 284. Ce mécanisme est au contraire bel et bien présent dans le raisonnement de Turgot car, lorsque ce dernier envisage le profit comme un «tout », il établit une hiérarchie détaillée de la grandeur de son taux et ce, d'après la nature de l'entreprise dans laquelle ce revenu intervient. Voir Turgot (1766), t. II, p. 591. D'autre part, Turgot intègre parfois l'amortissement du capital dans la rétribution de l'entrepreneur, ce que ne fait jamais Condorcet. Sur ce second point, voir ibid., p. 570 \& 591.

51 Au paragraphe LXIV des Réflexions, Turgot ne comprend, dans la rémunération de l'entrepreneur, que la récupération de ses avances et une somme correspondant au profit résultant de leur investissement dans une
} 
toutefois allusion en expliquant que l'intérêt des avances est déterminé par rapport au revenu résultant d'une entreprise moins risquée sollicitant un travail nul ou du moins peu conséquent (Turgot, 1767, t. II, p. 649-650. Voir aussi Turgot, 1770, t. III, p. 292). En tout état de cause, lorsque le risque se trouve évoqué, il est la plupart du temps dilué dans la rubrique salaire et travail de l'entrepreneur (Turgot, 1766, t. II, p. 569, 570, 574). En revanche, nous avons vu que selon Condorcet, il s'agissait non seulement pour l'entrepreneur de récupérer l'intérêt de ses avances, mais également de bénéficier d'une certaine somme en fonction de la gravité des divers dangers auxquels il se trouve exposé, ce dernier revenu étant de surcroît considéré, de façon systématique, indépendamment de la rémunération associée à son activité laborieuse. A cet égard, si certains commentateurs (R. L. Meek, 1973 ; P. Fontaine, 1989, p. 132 et suiv.) relèvent la filiation Cantillon-Turgot quant à la représentation d'un entrepreneur agissant en situation d'incertitude, l'emprise de la pensée de d'Alembert sur celle de Condorcet a mené ce dernier à une prise en compte nettement plus fructueuse de cette catégorie du risque.

Qu'en est-il maintenant de la théorie du profit de Condorcet si on la compare brièvement à celle de Smith ? Tout en faisant dépendre, comme Condorcet, le profit de la grandeur des valeurs accumulées, l'auteur de la Richesse des nations (1776) distingue clairement celui-ci du salaire lié au «travail d'inspection et de direction » (Smith, 1776, vol. I, p. 54-55), ce qui n'est pas le cas de Condorcet. En outre, Smith sépare, comme Condorcet (et à la différence de Turgot), la prime de risque perçue par l'entrepreneur du prix de « la peine » ou du « mal» qui résulte de l'emploi de son capital (ibid., p. 113-114 ; vol. III, p. 1025). Dans cette optique, il explique que les variations du profit sont dues, outre à «l'agrément ou au désagrément » résultant de l'emploi du capital, à « la sécurité » ou au « risque » (ibid., vol. I, p. 129) qui accompagne celui-ci. Ce dernier déterminant est même présenté comme «la probabilité ou non [de] réussir» (ibid., p. 116) dans telle ou telle entreprise. Néanmoins, cet usage du langage probabiliste demeure très allusif, pour ne pas dire métaphorique. En la matière, conclure à une influence de Smith sur Condorcet serait donc pour le moins dénué de fondements et ce d'autant plus que la nécessité d'établir divers seuils de probabilités afin de guider la décision est également mis en avant par Condorcet lorsqu'il s'agit de traiter des questions de caractère politique, comme par exemple celle d'étendre ou de restreindre les droits naturels de l'individu (voir notamment le Discours préliminaire de l'Essai, 1785, OB, p. 22-23). 
Bref, il paraît que ce sont, avant tout, les doutes de d'Alembert sur la capacité de la règle de l'espérance mathématique à se trouver en phase avec le comportement raisonnablement prudent des individus qui ont mené Condorcet à une analyse relativement fine $\mathrm{du}$ risque économique. Reste à présent à montrer que cette analyse de la conduite économique en univers incertain est de nature empirique. Pour ce faire, il est nécessaire de lever l'hypothèse selon laquelle les probabilités de perte et de gain, ainsi que les divers seuils de probabilités qui interviennent dans l'argumentation du marquis, sont supposés être connus. Comme nous l'avons déjà précisé, nous ne faisons en cela que suivre la démarche adoptée par Condorcet lui-même.

\section{4) Une analyse empirique du comportement économique en univers risqué}

C'est lorsque Condorcet traite du comportement de l'assureur qu'il plaide avec le plus d'insistance pour la collecte d'un certain nombre d'éléments concrets. Mais nous avons vu que l'entrepreneur fonde ses spéculations d'après les mêmes critères que l'assureur dans le raisonnement de Condorcet, à savoir l'espérance mathématique de son profit et un certain nombre de niveaux de probabilités, plus ou moins élevés selon l'amplitude du risque encouru. De ce point de vue, l'examen du comportement de l'assureur renvoie aussi à l'attitude de l'entrepreneur.

Commençons par envisager la détermination des différents degrés de probabilités, chacun fonction de la gravité des risques encourus par l'assureur. Recourir alors aux faits apparait, de prime abord, relativement complexe puisque les agents économiques sont considérés comme bornés et dirigés par leurs passions selon Condorcet (sur ce point, voir supra, p. 18-19). Mais il demeure, d'après lui, possible de se fonder sur l'observation du comportement d' « hommes sages », comportement pour ainsi dire idéal-typique. Dans son article Assurances maritimes (1784), Condorcet précise que cette observation peut prendre deux modalités différentes. La première consiste à déterminer ces différents risques en les rapprochant de ceux qui peuvent être considérés comme négligeables « dans la conduite de la vie ». On retrouve ici une idée exprimée par Condorcet dans d'autres textes, notamment dans le Discours préliminaire de l'Essai (1785), un risque négligeable étant parfois assimilé à celui qu'un ou plusieurs hommes «prudents», « sage et éclairé » ou encore «de bon sens » ignorent dans leur conduite quotidienne. Cela étant, cette première modalité ne dit rien sur 
le(s) critère(s) de choix permettant d'affirmer que tel ou tel assureur se comporte raisonnablement. En revanche, la seconde modalité que Condorcet envisage est nettement plus stimulante car elle véhicule un critère de choix clair et précis : le mécanisme de la concurrence. Plus précisément, l'estimation des divers seuils de probabilités, afférents à la plus ou moins grande dangerosité des risques encourus, peut résulter de l'observation directe des assureurs, prudents et sages, dont les opérations ont été, en quelque manière, positivement sanctionnées par la procédure du marché ; ces derniers ayant eux-mêmes calqué leur comportement sur celui pratiqué dans les bureaux d'assurance ayant prospéré. Ainsi, il s'agira « de savoir comment dans la pratique les hommes qui passent pour sages, et dont les projets ont réussi, ont résolu le même problème ; par exemple, quelle a été la probabilité de ne pas perdre que les assureurs ont su se procurer dans les différents bureaux d'assurances qui ont pu continuer le commerce avec avantage » (BC, p. 492, nos italiques) - et il va sans dire que les diverses primes de risque exigées par les assureurs varieront ensuite d'après les propres fonds dont ils disposent.

Il convient ensuite de déterminer la probabilité de gain - et inversement, celle de perte - intervenant dans l'espérance mathématique de l'assureur. Eu égard à l'assurance d'un commerce maritime, il faut ainsi établir la probabilité d'arrivée à bon port - et son contraire, celle de naufrage - des différents navires assurés. Les lettres $g$ et $p$ désignant respectivement la probabilité pour chaque navire d'arriver à bon port et celle de faire naufrage, Condorcet précise à ce sujet que « si $N$ est le nombre de vaisseaux perdus, par exemple, \& $M$ celui des vaisseaux qui n'ont point péri, on pourra sans une grande erreur, supposer pour le nombre $n$ des vaisseaux à assurer $g=\frac{M+1}{M+N+2}, p=\frac{N+1}{M+N+2}$, pourvu que $n$ soit beaucoup plus grand que $M+N »$ (ibid., p. 491) - la conformité de ces probabilités avec la réalité étant de plus en plus prononcée à mesure que le nombre des événements passés qui servent à leur évaluation est élevé. On reconnaît ici les formules issues du théorème dit de Bayes-Laplace, appui scientifique de la théorie du motif de croire de Condorcet. On s'en doute, cette théorie a des attaches empiriques car elle se fonde sur l'observation des événements passés. Condorcet précise d'ailleurs un peu plus loin : «on déterminera $g$ et $p$ d'après les événements d'un commerce semblable, antérieurs à l'époque qu'on voudrait considérer » (ibid., p. 492).

Bref, Condorcet écarte sa démonstration de toute gangue déductive. Premièrement, l'observation des événements passés permet de déterminer les probabilités de gain et de perte. Deuxièmement, la fortune initiale des agents, leur mise et les gains dont ils sont susceptibles 
de bénéficier sont estimés d'après leur valeur mathématique - et non morale et hypothétique comme chez D. Bernoulli - et mis en relation grâce à une interprétation statistique de l'espérance assortie de plusieurs niveaux de probabilité eux-mêmes tirés de l'observation. Aussi la théorie probabiliste du comportement économique de Condorcet répond-elle aux préoccupations de d'Alembert, ce dernier estimant que l'art de conjecturer appartient au champ des mathématiques non pas pures mais mixtes, c'est-à-dire appliquées aux phénomènes réels 52 .

\section{CONCLUSION}

Partir en quête des influences éventuelles de d'Alembert sur la pensée économique de Condorcet aurait pu paraître, à première vue, une entreprise illusoire. Pour s'en convaincre, il aurait suffi, d'une part, d'invoquer le fait que d'Alembert n'a pas écrit un seul texte à proprement parler économique 53 et de renvoyer, d'autre part, aux essais de synthèse des idées économiques de Condorcet (à savoir, principalement, F. Alengry (1904), p. 687-728 ; L. Cahen (1904), p. 9-24 ; E. Caillaud (1908) ; H. Delsaux (1931), p. 163-176 \& 207-214 ; J. S. Schapiro (1934), chpt VIII et, plus près de nous, J. C. Perrot (1988), G. Faccarello (1989), (1990), (1992)), ces études relevant l'influence d'écrivains tels que Quesnay, Turgot ou Smith et non celle de d'Alembert. Nous avons cependant tâché de montrer que l'influence de ce dernier sur Condorcet est, en la matière, considérable. Plus précisément, la conception mixte des sciences mathématiques et les réflexions probabilistes de d'Alembert rendent intelligibles l'application, par Condorcet, de l'art de conjecturer non seulement aux jeux de hasard ou aux assurances, comme le font certains de ses contemporains, mais également à n'importe quel type d'entreprise économique, qu'il soit de culture, de commerce ou d'industrie. A cet égard, il serait tentant d'attribuer à Condorcet le titre de fondateur de la théorie du choix économique

\footnotetext{
52 Dans plusieurs autres textes, Condorcet réitère le souhait de partir des faits tirés de l'expérience afin d'appréhender le comportement économique et les données à partir desquelles il doit s'établir. Voir Demande de renseignements dans différents ports (vers 1780), MS 857, f. 204 ; BC, p. 476 ; Précisions sur le programme du prix relatif aux assurances maritimes (vers 1783), BC, p. 468 ; Lettre de Bordeaux, 23 juin 1785 ; BC, p. 469471 ; Réponse à la lettre de Bordeaux, 11 juil. 1785 ; BC, p. 471-474 ; Tableau général (1793), OC, t. I, p. 567.

53 Nous ne prenons pas ici en compte plusieurs contributions, plus « techniques » qu'économiques, consacrées à des calculs d'annuités. Voir d'Alembert (1751 b), (1751 c), (1755), (1765) et (1783 a).
} 
en univers incertain. Il convient, quoi qu'il en soit, de relever la nature explicite et aboutie de la formalisation du facteur risque dans sa théorie du profit.

Le fait que ces analyses de Condorcet soient semble-t-il passées inaperçues donne à réfléchir. Il est vrai que plusieurs de ses travaux manuscrits n'ont été édités que tout récemment. Mais si ces textes permettent d'opérer des retranchements, d'étayer certains points et d'apporter même quelques éléments nouveaux, il n'empêche que le corpus déjà édité contient, peu ou prou, la définition particulière du profit livrée par Condorcet ainsi que les traits essentiels de sa théorie du comportement économique en avenir risqué. Seulement, la plupart des écrits alors sollicités ne figure pas dans l'édition de ses Euvres dites complètes, parue en 1847-49. De surcroît, l'objet principal de ces textes n'est pas économique mais mathématique - nous pensons en particulier au Mémoire sur le calcul des probabilités (1784) et aux Eléments (1786-87). Or, si les passages relatifs à l'économie que l'on y trouve sont pris isolément, sans être mis en rapport, non seulement avec l'ensemble de l'ouvrage mais aussi et surtout avec certaines analyses de d'Alembert, leur pleine intelligibilité est pour le moins difficile.

Par là même, de tels passages risquent d'être sous-estimés si on les compare à d'autres textes de Condorcet où se trouve, apparemment, récusée l'idée d'user d'une formalisation abstraite afin d'appréhender le comportement économique. A ce sujet, nous avons évoqué les fameuses Lettres à Verri à l'occasion desquelles Condorcet déclare que «l'envie d'acheter et celle de vendre ne sont susceptibles d'aucun calcul ». Plus de vingt ans après, il semblerait ne pas avoir changé d'avis lorsqu'il épingle, dans son Tableau général (1793), le traitement mathématique des opérations économiques consistant à « suppos[er] un ordre, une régularité, dont elles [ne sont] pas susceptibles » (OC, t. I, p. 567).

Entre-temps, Condorcet aura toutefois répondu aux doutes de d'Alembert. Et, tout en refusant de recourir, sous l'influence de son maître, à une démarche hypothétique afin de décrire le comportement des agents intervenant dans la sphère économique, il aura, dans les années 1780 , tenté de construire un cadre formel, aux attaches néanmoins empiriques, afin d'appréhender un tel comportement. A ce titre, ne pourrait-on pas affirmer que la conduite du négociant, c'est-à-dire d'une certaine manière son «envie de vendre », est finalement devenue, sous la plume de Condorcet, précisément «susceptible d'un calcul»? Pas réellement, car la vision qu'il développe alors du commerçant est celle d'un individu avançant des capitaux, à l'exemple de l'entrepreneur de culture ou d'industrie, non celle d'un offreur 
proprement dit. Par conséquent, il s'agirait plutôt de «l'envie d'investir», en vue d'un certain profit, qui serait en réalité devenue « susceptible d'un calcul». A cette occasion, c'est la prise en compte extrêmement fine du facteur risque, intégré dans l'analyse des spéculations de l'entrepreneur comme dans la définition du revenu qu'il perçoit, qui fait l'originalité du discours de l'académicien. Et la pensée de son mentor en mathématique, d'Alembert, constitue pour ainsi dire la boussole permettant de repérer cette contribution de Condorcet à l'analyse économique. 


\section{REFERENCES BIBLIOGRAPHIQUES}

\section{* Abréviations utilisées dans le cours du texte ou de la bibliographie}

- « OC » : Euvres de Condorcet, F. Arago et A. O’Connor (eds), Paris, Firmin-Didot, 184749.

- « BC » : Condorcet. Arithmétique politique. Textes rares ou inédits (1767-1789), B. Bru \& P. Crépel (eds), Paris, INED, 1994. Seules les références aux textes de Condorcet - et non aux commentaires de B. Bru \& P. Crépel - sont indiquées par cette abréviation.

- « OB » : Condorcet. Sur les élections et autres textes, O. de Bernon (ed.), Paris, Fayard, 1986.

\section{CONDORCET}

(Les cotes MS et $\mathrm{Z}$ désignent respectivement les manuscrits de Condorcet conservés à la Bibliothèque de l'Institut et au Bureau des Longitudes)

- « Arithmétique politique », [Fragments d'un premier projet d'encyclopédie], 1768, MS 874, f. 297 (extrait) ; BC, p. 228.

- [Première ébauche d'essai sur les probabilités], 1770, MS 883, f. 216-221 ; Z 30 f. 1-6 ; MS 875, f. 132-133 ; BC, p. 247-263.

- [Application des principes aux jeux de hasard et aux événements de la vie], vers 1770, MS 875, f. 116-125, 115 ; BC, p. 264-273.

- [Détermination de la mise d'un joueur], vers 1770, MS 875, f. 110-112 ; BC, p. 274-276.

- [Faire entrer dans le calcul l'effet des événements], vers 1770, MS 865, f. 84-89 ; BC, p. 295-300.

- Lettre de Condorcet à Piétro Verri, 7 nov. 1771, BC, p. 69-72.

- Lettre de Condorcet à Piétro Verri, début 1772, BC, p. 72-74.

- «Arithmétique politique », [Projet d'Abrégé de l'Encyclopédie], 1772, MS 873, f. 66-67 ; BC, p. 303.

- Arithmétique politique ou application des mathématiques aux sciences économiques, 1772, MS 855, f. 177-179; BC, p. 338-341.

- [Petit ouvrage «plus métaphysique que mathématique» sur le calcul des probabilités], vers 1773-74, MS 875, f. 84-109 ; BC, p. 283-294.

- Eloge de la Condamine, 1774, OC, t. II, p. 156-206.

- Réflexions sur l'arithmétique politique, vers 1780, fonds privé ; BC, p. 336-338.

- [Demande de renseignements dans différents ports, à propos des assurances maritimes], vers 1780, MS 857, f. 204-206 ; BC, p. 476.

- Eloge de Lieutaud, 1781, OC, t. II, p. 391-410.

- Eloge de Tronchin, 1781, OC, t. II, p. 498-513.

- Eloge de D. Bernoulli, 1782, OC, t. II, p. 545-580.

- Précisions sur le programme du prix relatif aux assurances maritimes, vers 1783, MS 857, f. 202-203 ; BC, p. 467-469.

- Eloge de d'Alembert, 1784, OC, t. III, p. 51-110.

- « Arithmétique politique », Encyclopédie méthodique, 1784, t. I, p. 135-136 ; BC, p. 483485.

- «Assurances maritimes », Encyclopédie méthodique, 1784, t. I, p. 150-154 ; BC, p. 485494. 
- « Probabilité », Encyclopédie méthodique, 1785, t. II, p. 649-663 ; BC, p. 494-515.

- Essai sur l'application de l'analyse à la probabilité des décisions rendues à la pluralité des voix, Paris, Imprimerie Royale, 1785 ; Chelsea, 1972.

- Discours préliminaire de l'Essai sur l'application de l'analyse à la probabilité des décisions rendues à la pluralité des voix, Paris, Imprimerie Royale, 1785 ; OB, p. 7-177.

- «Lettre de Bordeaux pour calculer les assurances contre les intempéries dans le domaine agricole », Journal de Paris, 23 juin 1785, p. 720-721 ; BC, p. 469-471.

- «Réponse à la lettre de Bordeaux », Journal de Paris, 11 juil. 1785, p. 796-797 ; BC, p. 471-474.

- Notes sur la thèse de Nicolas Bernoulli, 1785-86, MS 884, f. 1-2 ; MS 875, f. 179-185, 190 ; BC, p. 575-594.

- « Mémoire sur le calcul des probabilités », en six parties, Mémoires de l'Académie royale des sciences (de Paris) pour 1781 (1784), p. 707-728; pour 1782 (1785), p. 674-691 ; pour 1783 (1786), p. 539-559 ; pour 1784 (1787), p. 454-468 ; BC, p. 385-448.

- Eléments du calcul des probabilités et son application aux jeux de hasard, à la loterie et aux jugements des hommes, 1786-87, Paris, Fayolle, 1805 ; OB, p. 483-596.

- Eloge de Bouvart, 1787, OC, t. III, p. 272-293.

- Essai sur la constitution et les fonctions des assemblées provinciales, 1788, OC, t. VIII, p.115-659.

- Sur la forme des élections, 1789, OC, t. IX, p. 285-330.

- Tableau général de la science qui a pour objet l'application du calcul aux sciences politiques et morales, 1793, OC, t. I, p. 539-573.

\section{D'ALEMBERT}

- Encyclopédie ou Dictionnaire raisonné des sciences, des arts et des métiers, D. Diderot \& J. R. d'Alembert (eds.), 1751-65 ; New York, Reader Microprint Corporation, 1969, 5 vol, 35 t.

- « Absent », dans J. R. d’Alembert \& D. Diderot (eds), 1751 a, vol. I, t. 1, p. 40.

- « Annuité », 1751 b, dans J. R. d'Alembert \& D. Diderot (eds), vol. I, t. I, p. 484-486.

- « Arrérages », 1751 c, dans J. R. d'Alembert \& D. Diderot (eds), vol. I, t. I, p. 705-707.

- « Croix ou Pile », dans J. R. d'Alembert \& D. Diderot (eds), 1754, vol. I, t. 4, p. 512-513.

- « Escompte », 1755, dans J. R. d'Alembert \& D. Diderot (eds), vol I, t. 5, p. 944-945.

- « Géomètre », dans J. R. d'Alembert \& D. Diderot (eds), 1757, vol. II, t. 7, p. 627-629.

- Essai sur les éléments de philosophie ou sur les principes des connaissances humaines, 1759 ; Paris, Fayard, 1986, p. 9-190.

- "Réflexions sur le calcul des probabilités », 1761 a, Opuscules mathématiques, Paris, David, 1761, vol. II, p. 1-25.

- «Sur l'application du calcul des probabilités à l'inoculation de la petite vérole », $1761 \mathrm{~b}$, Opuscules mathématiques, Paris, David, 1761, vol. II, p. 26-95.

- « Intérêt », dans J. R. d'Alembert \& D. Diderot (eds), 1765, vol. II, t. 8, p. 819-823.

- «Doutes et questions sur le calcul des probabilités », 1767 a, Euvres complètes de d'Alembert, Paris, Belin, 1821, t. I, p. 451-462.

- « Réflexions sur l'inoculation », 1767 b, Euvres complètes de d'Alembert, Paris, Belin, 1821, t. I, p. 463-514.

- «Extraits de plusieurs lettres de l'auteur sur différents sujets, écrites dans le courant de l'année 1767. V : sur le calcul des probabilités (1768 a, p. 73-79). VI : sur l'analyse des jeux (1768 b, p. 80-92). VIII : sur un mémoire de Bernoulli concernant l'inoculation (1768 c, p. 98-105) », Opuscules mathématiques, vol. IV, Paris, Briasson, 1768. 
- « Extraits de lettres sur le calcul des probabilités, et sur les calculs relatifs à l'inoculation. I : sur le calcul des probabilités (1768 d, p. 283-310). II : sur les calculs relatifs à l'inoculation (1768 e, p. 310-341), Opuscules mathématiques, vol IV, Paris, Briasson, 1768.

- «Sur le calcul des probabilités », 1780, Opuscules mathématiques, vol. VII, Paris, Jombert, 1780 .

- « Sur les annuités », 1783 a, Opuscules mathématiques, 1783, vol. IX, MS 1793, f. 1-161 (cote de la Bibliothèque de l'Institut).

- "Sur l'application du calcul des probabilités à certaines questions », 1783 b, Opuscules mathématiques, vol. IX, 1783, MS 1793, f. 369-387 (cote de la Bibliothèque de l'Institut).

\section{AUTRES AUTEURS}

- ALENGRY F., Condorcet, Guide de la Révolution française, Paris, 1904 ; Genève, Slatkine, 1971.

- ALLAIS M., «Le comportement de l'homme rationnel devant le risque : critique des postulats et axiomes de l'école américaine», Econometrica, vol. 21, n¹, oct. 1953, p. 503546.

- ALLAIS M., «The foundations of a positive theory of choice involving risk and a criticism of the postulates and axioms of the american school », 1979 a, dans M. Allais \& O. Hagen (1979), p. 27-145.

- ALLAIS M., "The so-called Allais Paradox and rational decisions under uncertainty », 1979 b, dans M. Allais \& O. Hagen (1979), p. 437-681.

- ALLAIS M. \& HAGEN O. (sous la dir. de), Expected utility hypotheses and the Allais paradox, Dordrecht, D. Reidel Publishing Company, 1979.

- ARROW K. J., Social choice and individual values, New York, 1951 (1 $^{\text {ère }}$ ed.), 1963 (2 ed.), trad. Choix collectifs et préférences individuelles, Paris, Calmann-Lévy, 1974.

- ARROW K. J., " Formal Theories of social welfare », P. Wiener (ed.), Dictionary of the history of ideas, vol. 4, 1973, p. 276-284.

- BAKER K. M., «Un 'éloge' officieux de Condorcet : sa notice historique et critique sur Condillac », Revue de synthèse, III ${ }^{\mathrm{e}}$ série, n47-48, juil.-déc. 1967, p. 227-244.

- BAKER K. M., Condorcet. From natural philosophy to social mathematics, Chicago, University of Chicago Press, 1975 ; trad. de M. Nobile : Condorcet, raison et politique, Paris, Hermann, 1988.

- BEARD R., PENTIKAINEN T. \& PESONEN E., Risk theory - The stochastic basis of insurance, 1969, $3^{\mathrm{e}}$ ed. : 1984, London \& New York, Chapman \& Hall (eds).

- BERNOUlli D., BERNOUlli N., CRAMER G., MONTMORT P. R. de, «Correspondance », 1713-1732 ; Die werke von Jakob Bernoulli, t. III, Bâle, Birkhäuser, 1975, p. 557-567.

- BERNOULLI D., « Specimen theoriae novae de mensura sortis », Commentarii Academiae Scientiarum Imperialis Petropolitanae, 1738, t. V, p. 175-192 ; trad. de R. Charreton : «Esquisse d'une théorie nouvelle de mesure du sort », Cahiers du séminaire d'histoire des mathématiques, 6, 1985, p. 61-77.

- BERNOULLI J., « Meditationes », 1677-1705, Die werke von Jakob Bernoulli, t. III, Bâle, Birkhäuser, 1975, p. 21-89.

- BERNOULLI J., «Ars conjectandi », 1713, Die werke von Jakob Bernoulli, t. III, Bâle, Birkhäuser, 1975, p. 107-286 ; trad. bilingue de la $4^{\mathrm{e}}$ partie par N. Meusnier (1987).

- BERNOULLI N., De usu artis conjectandi in jure, 1709 ; trad. bilingue de N. Meusnier : L'usage de l'art de conjecturer en droit, Séminaire d'histoire des probabilités et de la statistique, Université Paris VIII, 1992. 
- BERNOULLI N., «Lettre à Cramer », 3 juil. 1728, dans D. Bernoulli et allii (1713-1732), p. 562-563.

- BICQUILLEY C. F., Théorie élémentaire du commerce, Toul, Vve Carez, an XII (1804) ; rééd. critique et commentée par P. Crépel, Lyon, Aléas, 1995.

- BLACK D., The theory of committes and elections, Cambridge University Press, 1958.

- BORCH K., "The theory of risk», Journal of the royal statistical society, Séries B (methodological), vol. 29, n³, 1967, p. 432-467.

- BORCH K., "Decision rules depending on the probability of ruin », Oxford economic papers, 20, $\mathrm{n}^{\circ} 3$, nov. 1968, p. 1-10.

- BRU B., « Doutes de d'Alembert sur le calcul des probabilités », 1983, dans J. Roger \& allii (1989), p. 279-302.

- BRU B., « Statistique et bonheur des hommes », Revue de synthèse, IV ${ }^{\mathrm{e}}$ série, $\mathrm{n}^{\circ} 1$, janv.mars 1988, p. 69-95.

- BRU B. \& CREPEL P., Condorcet. Arithmétique politique. Textes rares ou inédits (17671789), Paris, INED, 1994.

- BRU B., "Condorcet, Mathématique sociale et vérité », Mathématiques, informatique et sciences humaines, ${ }^{\circ} 128,1994$, p. 5-14.

- BUFFON G. L., « Lettre à Cramer », 1730, dans G. L. Buffon (1777), p. 49.

- BUFFON G. L., Essai d'arithmétique morale, Paris, 1777, rééd. par J. L. Binet \& J. Roger dans Un autre Buffon, Paris, Hermann, 1977, p. 32-91.

- BÜHLMANN H., Mathematical methods in risk theory, Springer Verlag, 1970.

- CAHEN L., Condorcet et la Révolution française, Paris, 1904 ; Genève, Slatkine, 1970.

- CAILLAUD E., Les idées économiques de Condorcet, Poitiers, Imprimerie Bousrez, 1908.

- CANARD N. F., Principes d'économie politique, Paris, F. Buisson, 1801.

- COUMET E., « La théorie du hasard est-elle née par hasard ?», Annales E. S. C., n³, 1970, p. 574-598.

- CRAMER G., «Lettres à N. Bernoulli », 21 mai et 27 sept. 1728, dans D. Bernoulli et allii (1713-1732), p. 560-562 \& 563-564.

- CRAMER H., «Collective risk theory : a survey of the theory from the point of view of the theory of stochastic process ", 7th jubilee volume of skandia insurance company stockholm, 1955, p. 51-92, dans Harald Cramér, Collected works, Anders Martin-Löf (ed.), Berlin, Heidelberg, New York, Springer Verlag, vol. II, p. 1028-1114.

- CREPEL P., « Condorcet, la théorie des probabilités et les calculs financiers », 1988 a, dans R. Rashed (1988), p. 267-325.

- CREPEL P., «Condorcet et l'estimation statistique », Journal de la Société de Statistique de Paris, t. 129, n¹-2, 1988 b, p. 46-67.

- CREPEL P., « Les calculs économiques et financiers de Condorcet pendant la Révolution », 1990, dans G. Faccarello \& P. Steiner (1990), p. 339-350.

- DASTON L. J., «D'Alembert's critique of probability theory », Historia mathematica, 6, 1979, p. 259-279.

- DASTON L. J. , Classical probability in the enlightenment, Princeton University Press, 1988.

- DELQUIE P., Eliciting preferences free of compatibility and prominence biaises, Note de recherche, GRID, ENS Cachan, août 1993.

- DELSAUX H., Condorcet journaliste (1790-1794), Paris, Honoré Champion, 1931.

- DIDEROT D., «Absent » - ajout à l'article de d'Alembert (1751 a), vol. I, t. 1, p. 40-41.

- DUBOURDIEU J., Théorie mathématique du risque dans les assurances de répartition, Paris, Gauthier-Villars, 1952. 
- DUTKA J., « On the Saint Petersburg Paradox », Archive for the history of exact sciences, 39, 1988, p. 13-39.

- FACCARELLO G., «Condorcet : au gré des jugements »: Présentation de la troisième partie (Economie), dans P. Crépel \& C. Gilain (sous la dir. de), Condorcet - Mathématicien, économiste, philosophe, homme politique, Actes du Colloque International Condorcet, juin 1988, Paris, Minerve, 1989, p. 121-149.

- FACCARELLO G. \& STEINER P. (sous la dir. de), La pensée économique pendant la Révolution Française, P. U. G., 1990.

- FACCARELLO G., «Le legs de Turgot : aspects de l'économie politique sensualiste de Condorcet à Rœderer », dans G. Faccarello et P. Steiner (1990), p. 67-107.

- FACCARELLO G., « Turgot et l'économie politique sensualiste », dans A. Béraud et G. Faccarello (sous la dir. de), Nouvelle histoire de la pensée économique, t. I, Paris, La Découverte, 1992, p. 254-288.

- FELLER W., An introduction to probability theory and its applications, $2^{\mathrm{e}}$ ed., New York \& London, J. Wiley (ed.), 1966, vol. 2.

- FINETTI B. de, « Il problema dei "peini" », Giornale dell'istituto italiano degli attuari, 1, 1940, p. 1-88.

- FONTAINE P., L'entrepreneur chez Turgot, Thèse pour le doctorat de troisième cycle, Université de Paris I, 1989.

- FRECHET M., Les mathématiques et le concret, Paris, P.U.F., 1955.

- FRISI P., « Notes et appendice» de P. Verri (1772).

- GRANGER G. G., La mathématique sociale du marquis de Condorcet, 1956 ; Paris, Odile Jacob, 1989.

- 's GRAVESANDE G. J., Introduction à la philosophie, 1736 ; Paris, Lyon, Périsse Frères, 1842.

- GUILBAUD G. T., «Les théories de l'intérêt général et le problème logique de l'agrégation », Economie appliquée, 5, n4, 1952, p. 501-584.

- HACKING I., The emergence of probability, Cambridge University Press, 1975.

- HALD A., A history of probability and statistics and their applications before 1750, New York, Wiley, 1990.

- HANKINS T. L., Jean d'Alembert, Science and the Enlightenment, Oxford, Clarendon Press, 1970.

- HUME D., « De l'entendement », Traité de la nature humaine, Livre 1, 1739 ; Paris, GF, Flammarion, 1995.

- JORLAND G., «The Saint Petersburg Paradox 1713-1937 », The Probalistic Revolution, L. Kruger, L. J. Daston. \& M. Heidelberger (sous la dir. de), Massachusetts Institute of Technology, 1987, vol. 1, p. 157-190.

- KAHNEMAN D. \& TVERSKY A., "Prospect theory : an analysis of decision under risk », Econometrica, vol. 47, n², mars 1979, p. 263-291.

- KEYNES J. M., "A Treatise on Probability », 1921, The Collected Writings of John Maynard Keynes, vol. VIII, Macmillan Cambridge University Press, 1988.

- LACROIX S. F., Traité élémentaire du calcul des probabilités, Paris, $2^{\mathrm{e}}$ ed., 1822.

- LAPLACE P. S., Théorie analytique des probabilités, $3^{\mathrm{e}}$ ed., Paris, 1812, rééd. dans Euvres, Paris, Gauthiers-Villars, 1878-1912, vol. VII.

- LE BRAS H., « D'Alembert et la querelle de l'inoculation - Le sujet contre la population », dans J. Roger \& allii (1989), p. 293-302.

- LLOYD H., An essay on the theory of money, Londres, 1771.

- MacCRIMMON K. R. \& LARSSON S., "Utility theory : axioms versus "paradoxes" », dans M. Allais \& O. Hagen (1979), p. 333-409. 
- MASSE P., « Réflexions sur les comportements rationnels en économie aléatoire », Cahiers du séminaire d'économétrie, 1953, p. 11-58.

- MEEK R. L., "Adam Smith and the classical concept of profit », Scottish journal of political economy, vol. I, mars 1954, p. 138-153.

- MEEK R. L., "Introduction », Turgot on progress, sociology and economics, 1973, p. 1-33.

- MEUSNIER N., Jacques Bernoulli et l'Ars conjectandi, Université de Rouen Haute Normandie, IREM, 1987.

- MEUSNIER N., «Argumentation et démonstration : à quoi sert la démonstration de la "loi des grands nombres" de Jacques Bernoulli (1654-1705)», La démonstration mathématique dans l'histoire, Actes du $7^{\mathrm{e}}$ colloque Inter-IREM, Besançon, 12 \& 13 mai 1989, IREM de Besançon et de Lyon, 1990, p. 81-98.

- MEUSNIER N., « La passe de l'espérance - L'émergence d'une mathématique du probable au XVII ${ }^{\mathrm{e}}$ siècle », C.A.M.S., Série Histoire du calcul des probabilités, n²1, mai 1995.

- MORGENSTERN O., « Some Reflections on Utility », 1974, dans M. Allais \& O. Hagen (1979), p. 175-183.

- MORILHAT C., La prise de conscience du capitalisme. Economie et philosophie chez Turgot, Paris, Méridiens Klincksieck, 1988.

- MOULIN H. \& YOUNG H. P., " Condorcet, Marie Jean Antoine Nicolas Caritat, Marquis de », J. Eatwell, M. Milgate, P. Newman (eds), The new Palgrave, a dictionary of economics, $3^{\mathrm{e}}$ ed., vol. 1, Londres, MacMillan, 1987, réimpr. 1991.

- NANSON E. J., « Mémoire sur les méthodes d'élection », Transactions of the Royal Society of Victoria, Melbourne, vol. XIX, 1883, p. 197-240.

- PATY M., « La critique par d'Alembert des conditions d'une théorie des probabilités physiques », Fundamenta scientiae, vol. 8, n³-4, 1987, p. 257-282.

- PATY M., « D’Alembert et les probabilités », dans R. Rashed (1988), p. 203-265.

- PERROT J. C., «Condorcet : de l'économie politique aux sciences de la société », Revue de synthèse, $4^{\mathrm{e}}$ série, 1, janv.-mars 1988, p. 13-37.

- QUESNAY F., « Grains », 1757, François Quesnay et la Physiocratie, Paris, INED, 1958, t. II, p. 459-510.

- QUIGGIN J., "A theory of anticipated utility», Journal of economic behavior and organization, 3, 1982, p. 225-243.

- RASHED R. (ed.), Sciences à l'époque de la Révolution française, Paris, Blanchard, 1988.

- RAVIX J. T. \& ROMANI P. M., « Turgot et le concept classique de profit», Etudes d'économie classique et néo-ricardienne, 1982, p. 29-56.

- RAVIV A., "The design of an optimal insurance policy », The american economic review, vol. $69, \mathrm{n}^{\circ} 1$, mars 1979 , p. 84-96.

- ROGER J., «Commentaires » de Buffon (1777), 1977, p. 25-31.

- ROGER J., BELAVAL Y., PAPPAS J., TATON R., COUMET E. (sous la dir. de), Jean d'Alembert, savant et philosophe : portrait à plusieurs voix, Actes du Colloque du 15-18 juin 1983, Paris, Edition des Archives Contemporaines, 1989.

- SAMUELSON P. A., "Saint Petersburg Paradoxes : defanged, dissected, and historically described », Journal of economic literature, vol. XV, $n^{\circ} 1$, mars 1977, p. 24-55.

- SCHAPIRO J. S., Condorcet and the rise of liberalism, New York, Harcourt, Brace \& Co, 1934.

- SCHELLE G., Euvres de Turgot et documents le concernant, Paris, Félix Alcan, 1913-23, 5 t.

- SHEYNIN O. B., « Early history of the Theory of Probability », Archive for history of exact sciences, vol. 17, 1977, p. 201-259. 
- SMITH A., Enquête sur la nature et les causes de la richesse des nations, 1776 ; trad. de P. Taïeb, Paris, PUF, 5 vol., 1995.

- SWIJTINK Z. J., "D'Alembert and the maturity of chances », Studies in History and Philosophy of Sciences, vol. 17, n³, 1986, p. 327-349.

- TODHUNTER I., A history of the mathematical theory of probability - From the time of Pascal to that of Laplace, Cambridge et Londres, Mac Millan and co., 1865.

- TURGOT A. R. J., «Réflexions sur la formation et la distribution des richesses », 1766, dans G. Schelle (ed.), 1914, t. II, p. 533-601.

- TURGOT A. R. J., «Observations sur les mémoires récompensés par la Société d'Agriculture de Limoges : sur le mémoire de Saint-Peravy », 1767, dans G. Schelle (ed.), 1914, t. II, p. 641-658.

- TURGOT A. R. J., «Lettres au Contrôleur Général (abbé Terray) sur le commerce des grains », oct.-déc. 1770, dans G. Schelle (ed.), 1919, t. III, p. 265-354.

- TVERSKY A. \& KAHNEMAN D., "Advances in prospect theory : cumulative representation of uncertainty », Journal of risk and uncertainty, V, 1992, p. 297-323.

- VERRI P., Meditazioni sull'economia politica, $6^{\mathrm{e}}$ ed. italienne : 1772, Accresciulta dall'Autore, Livorno, Nella Stampera dell'Enciclopedia, avec notes et appendice de P. Frisi ; trad. par F. Neale (sans les notes et l'appendice de Frisi) : Méditations sur l'économie politique, Paris, Masson, 1823.

- YAARI M. E., « The dual theory of choice under risk », Econometrica, vol. 55, n¹, 1987, p. 95-115.

- YAMAZAKI E., «D'Alembert et Condorcet, quelques aspects de l'histoire du calcul des probabilités », Japanese Studies in the History of Science, 1971, p. 59-93. 